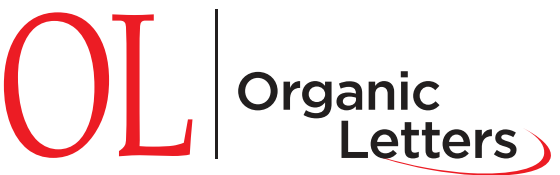

\title{
Harnessing Thiol as a Benzyl Reagent for Photocatalytic Reductive Benzylation of Imines
}

\author{
Jinghui Zhang and Muliang Zhang*
}

Cite This: https://dx.doi.org/10.1021/acs.orglett.0c00065

Read Online

ACCESS

Ш Metrics \& More

回 Article Recommendations

Supporting Information

$$
\text { Bn-SH }+\mathrm{R}^{2}=\mathrm{N}_{\mathrm{R}^{1}} \frac{\begin{array}{l}
\left.\operatorname{Ir}\left(\mathrm{dF}\left(\mathrm{CF}_{3}\right) \mathrm{ppy}\right)_{2}(\mathrm{dtbbpy})\right] \mathrm{PF}_{6}(1 \mathrm{~mol} \%) \\
\mathrm{Ph}_{3} \mathrm{P}(0.4 \mathrm{mmol}), \mathrm{TFA}(10 \mathrm{~mol} \%)
\end{array}}{\operatorname{DMA}(0.1 \mathrm{M}), \text { Blue LEDs, } \mathrm{rt}}
$$

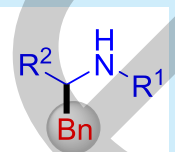

ABSTRACT: Photocatalytic reductive benzylation of imines with benzyl thiol provides an alternative to the substitution of traditional alkyl coupling partners. Benzyl thiols may be directly used as a benzyl reagent by phosphoranyl-assisted desulfurization, and this represents a new perspective in synthetic planning and implementation. The photoinduced $\alpha$-amino radical anions engage in subsequent $\mathrm{C}-\mathrm{C}$ coupling with benzyl radicals. Furthermore, a large gram-scale synthesis is demonstrated by utilizing continuousflow photomicroreactors.

$\mathrm{T}$ hiols are readily available, and a highly reactive thio group acts as a latent activating group to trigger different types of chemical reactions in organic synthesis. ${ }^{1}$ Over the past few decades, various radical-based types of transformations of thiols have been extensively developed by utilizing thiyl radical intermediates. ${ }^{2}$ For example, nature takes advantage of the thiyl radical in many enzymatic processes, including deoxygenation of ribonucleotides ${ }^{3}$ or the de novo preparation of DNA precursors. ${ }^{4}$ Not surprisingly, many elegant synthetic applications of thiyl radicals such as addition to unsaturated systems (olefins, alkynes, isonitriles, and thiocarbonyl groups), ${ }^{5}$ thiolene $^{6}$ or -yne type reactions, ${ }^{7}$ reductive processes, ${ }^{8}$ radical cascade cyclization reactions, ${ }^{9}$ addition-rearrangement, ${ }^{10}$ and cross-coupling reaction ${ }^{11}$ have been achieved (Scheme 1A). In

Scheme 1. Various Radical-Based Types of Transformation of Alkyl Thiols

A) The thiyl radicals enable C-S bond formation
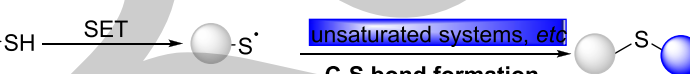

B) The HAT strategy with the thiyl radicals

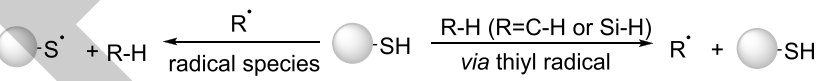

C) This work: Harnessing thiol as benzyl reagent for benzylation of imines

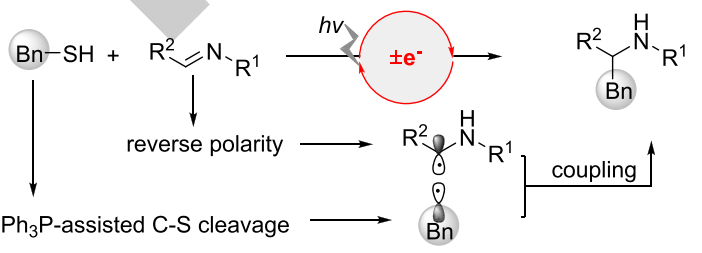

addition, thiols could also serve as hydrogen atom transfer (HAT) catalysts, $^{12}$ enabling the straightforward activation of the inert bonds of substrates $(\mathrm{C}-\mathrm{H}$ or $\mathrm{Si}-\mathrm{H})$ via the thiyl radical, ${ }^{13}$ or transfer a hydrogen atom to other radical intermediates to assist in the catalytic cycle ${ }^{14}$ (Scheme 1B).

Carbon-heteroatom bond cleavage/functionalization is synthetically valuable for manipulating molecules with sensitive functional groups, and selective chemical bond cleavage presents a new perspective on their application. ${ }^{15}$ In the past several decades, reductive desulfurization of thiol derivatives has been well established as a synthetic tool in organic synthesis, and its application is highlighted as carbon synthons by certain activating steps in which thiols could be converted into derivatives such as thioethers. ${ }^{16}$ However, current applications of radical-based conversions of thiols are restricted to carbon-sulfur formation, and alkyl thiols have yet to be widely recognized as direct alkylating agents. ${ }^{17}$ This concept was reported by the Nanni group in 2004; they achieved the direct desulfurizing radical addition to a large excess of olefins by utilizing tert-butyl isocyanide as a desulfurization reagent. ${ }^{17 a}$ We envisioned that the astute application of such a concept would allow a myriad of more valuable coupling reactions to be explored. Recently, visible light photoredox-catalyzed polarity reversal of imine through the proton-coupled electron transfer (PCET) manifold establishes a new umpolung pathway for synthesizing $\alpha$-functionalized amines by many research groups, ${ }^{18}$ providing many opportunities to discover a number of promising transformations. The key to success is the

Received: January 7, 2020 
generation of $\alpha$-amino radicals, which have been demonstrated to subsequently engage in radical addition or radical-radical coupling or to react with other electrophilic species. Inspired by these elegant works, we wondered whether a desulfurizing process could offer alkyl radical intermediates, which would further be utilized for subsequent carbon-carbon coupling with $\alpha$-amino radical anions generated by photoinduced reductive SET umpolung of imine (Scheme 1C). As a proof of concept, we herein report the first desulfurizing benzylation reaction of alkyl thiols with imine by visible light photoredox catalysis.

Our study began with optimization of the desulfurizing benzylation of benzyl thiol 2a with imine 1a (Table 1). The

Table 1. Optimization of the Reaction Conditions ${ }^{a}$

\begin{tabular}{|c|c|c|c|}
\hline \multirow{2}{*}{$\begin{array}{c}p \text {-tolyl } \backslash \mathrm{N} \\
0.2 \mathrm{mmol} \\
1 \mathrm{a}\end{array}$} & \multirow{2}{*}{$\begin{array}{c}+\mathrm{BnSH} \\
0.4 \mathrm{mmol} \\
2 \mathrm{a}\end{array}$} & $\begin{array}{c}\left.\operatorname{Ir}\left(\mathrm{dF}\left(\mathrm{CF}_{3}\right) \mathrm{ppy}_{2}\right)_{2}(\mathrm{dtbbpy})\right] \mathrm{PF}_{6}(1 \mathrm{~mol} \%) \\
\mathrm{Ph}_{3} \mathrm{P}(0.4 \mathrm{mmol}), \operatorname{TFA}(10 \mathrm{~mol} \%)\end{array}$ & \multirow{2}{*}{$\underbrace{p \text {-tolyl }}_{\substack{\mathrm{Bn} \\
\mathbf{3 a}}}$} \\
\hline & & DMA (0.1 M), Blue LEDs, rt & \\
\hline entry & \multicolumn{2}{|c|}{ variation of standard conditions } & yield (\%) \\
\hline 1 & \multicolumn{2}{|l|}{ none } & 80 \\
\hline 2 & \multicolumn{2}{|c|}{$t$-BuNC instead of $\mathrm{Ph}_{3} \mathrm{P}$} & not detected \\
\hline 4 & \multicolumn{2}{|c|}{ no $\mathrm{Ph}_{3} \mathrm{P}$} & not detected \\
\hline 5 & \multicolumn{2}{|c|}{ no TFA } & 45 \\
\hline 6 & \multicolumn{2}{|c|}{ AcOH instead of TFA } & 54 \\
\hline 7 & \multicolumn{2}{|c|}{ TsOH instead of TFA } & 58 \\
\hline 8 & \multicolumn{2}{|c|}{1 equiv of $2 \mathbf{a}$ was used } & 61 \\
\hline 9 & \multicolumn{2}{|c|}{ no light or no photocatalyst } & not detected \\
\hline
\end{tabular}

${ }^{a}$ Standard conditions: $1 \mathrm{a}(0.2 \mathrm{mmol}), 2 \mathrm{a}$ thiols $(0.4 \mathrm{mmol}, 2.0$ equiv), $\mathrm{Ph}_{3} \mathrm{P}(0.4 \mathrm{mmol}, 2.0$ equiv), TFA (10 mol \%), DMA $(2.0 \mathrm{~mL}), \mathrm{rt}, 5$ $\mathrm{W}$ blue light-emitting diodes (LEDs), $36 \mathrm{~h}$.

optimized reaction conditions include $1 \mathrm{~mol} \% \operatorname{Ir}\left[\mathrm{dF}\left(\mathrm{CF}_{3}\right)\right.$ ppy $]_{2}$ (dtbbpy) $\mathrm{PF}_{6}$ as the photocatalyst, 2.0 equiv of $\mathrm{Ph}_{3} \mathrm{P}$ as the sulfur atom transfer reagent, TFA as the additive, and DMA as the solvent (entry 1 ). The desired $\alpha$-benzyl amine product could been isolated in $80 \%$ yield after $36 \mathrm{~h}$ under visible light irradiation. When $t$-BuNC was used as the desulfurization reagent instead of $\mathrm{Ph}_{3} \mathrm{P}$, the reaction did not work at all (entry 2). If the reaction system lacked $\mathrm{Ph}_{3} \mathrm{P}$, the amine product was not obtained, showing $\mathrm{Ph}_{3} \mathrm{P}$ played an important role (entry 3). In addition, we also compared different additives, including $\mathrm{AcOH}$ and $\mathrm{TsOH}$. The reaction could also proceed smoothly, albeit with lower yields (entries $5-7$ ). When the amount of benzyl thiol (2a) was decreased to 1.0 equiv, the yield decreased (61\%, entry 8$)$. This is because some benzyl thiols could undergo a desulfurizing process to deliver the hydrodesulfurizing product. Finally, control experiments proved that the desulfurizing benzylation reaction could not occur in the absence of either light or a photocatalyst (entry 9).

With the optimized conditions in hand, we began to investigate the substrate scope for this desulfurizing benylation reaction. First, the scope with respect to alkyl thiol was explored in Scheme 2. It was found that benzyl radicals generated from alkyl thiols could react well with imine to deliver $\alpha$-benzylated amines in good yield. In general, alkyl thiol bearing both electron-rich (e.g., - $\mathrm{Me}$ and $-\mathrm{MeO}$ ) and electron-poor groups (e.g., $-\mathrm{F},-\mathrm{Cl}$, and $-\mathrm{Br}$ ) at the aromatics could proceed smoothly in $56-80 \%$ yield, providing different substituted $\alpha$-benzylated amines $(\mathbf{3 a}-\mathbf{3 f})$. The generated unsymmetrical secondary benzyl radical could tolerate the
Scheme 2. Substrate Scope for Alkyl Thiols ${ }^{a}$

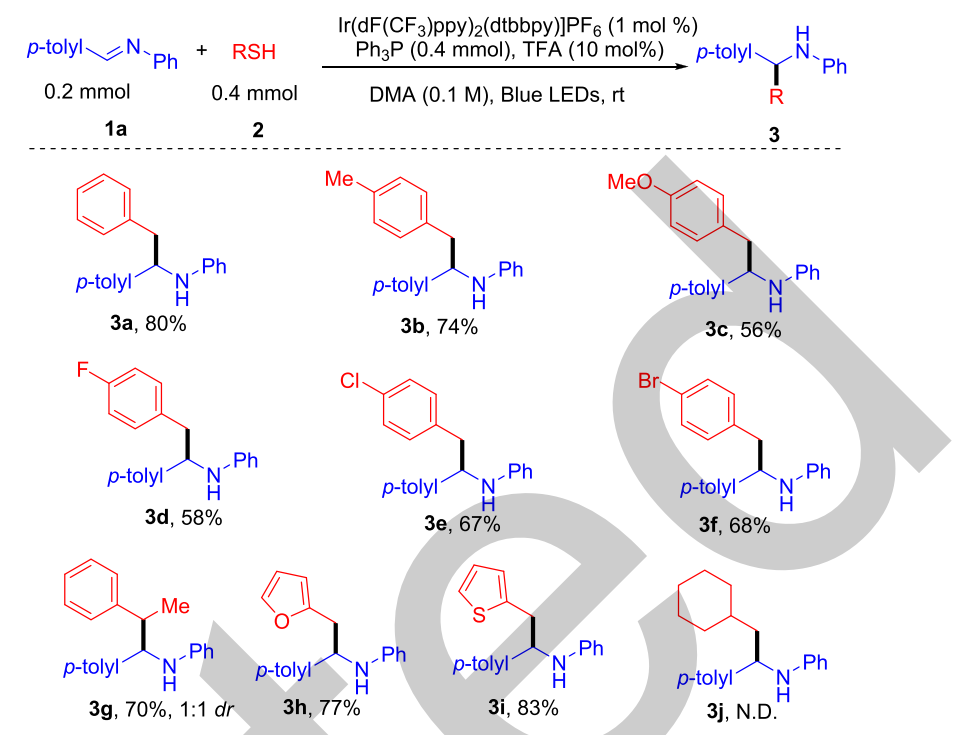

${ }^{a}$ Reaction conditions: photocatalyst $(1 \mathrm{~mol} \%)$, 1a imine $(0.2 \mathrm{mmol})$, 2 thiols ( $0.4 \mathrm{mmol}, 2.0$ equiv), $\mathrm{Ph}_{3} \mathrm{P}(0.4 \mathrm{mmol}, 2.0$ equiv), TFA ( 10 mol \%), DMA $(2.0 \mathrm{~mL}), \mathrm{rt}, 5 \mathrm{~W}$ blue LEDs, $24-36 \mathrm{~h}$.

conditions with high efficiency, though no diastereoselectivity was detected (3g). Heteroaromatic benzyl thiols, including furan and thiophene, uniformly underwent this desulfurizing benzylation, giving $\alpha$-heterocyclic amines products (3h and $3 \mathbf{i}$, respectively). However, non-benzyl alkyl thiol failed in this system (3j).

Subsequently, the substrate scope of imines was also explored, as shown in Scheme 3. When variations of the

Scheme 3. Substrate Scope for Imines ${ }^{a}$

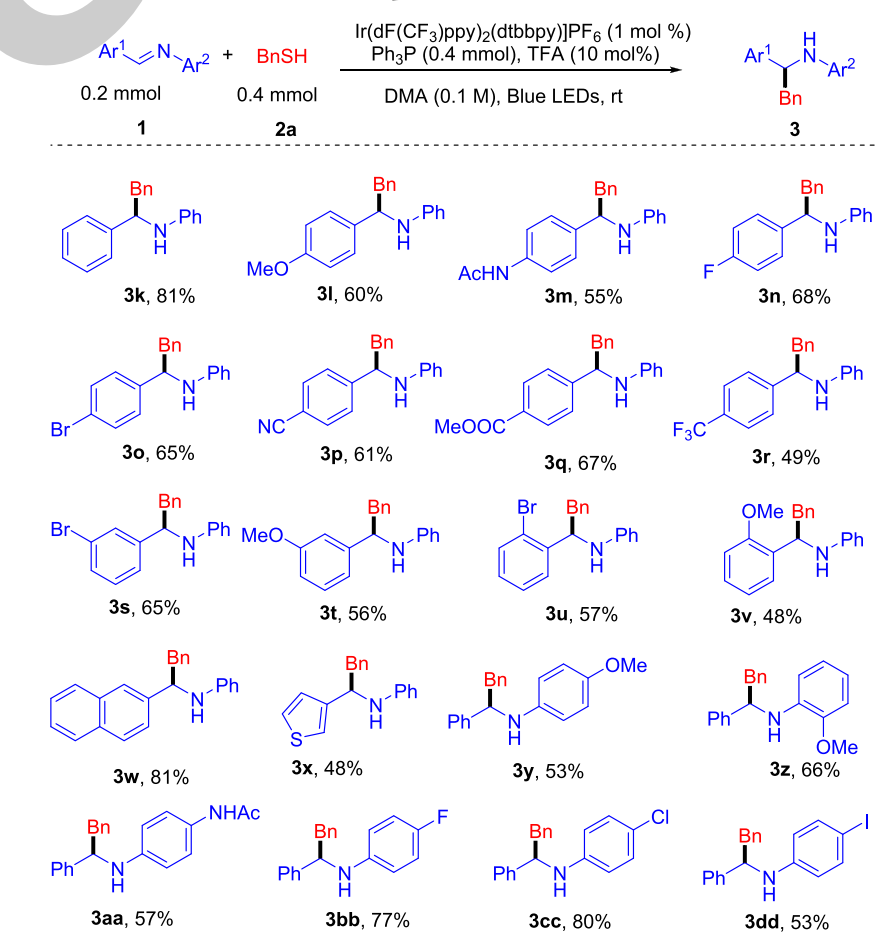

${ }^{a}$ Reaction conditions: photocatalyst $(1 \mathrm{~mol} \%), 1$ imine $(0.2 \mathrm{mmol})$, 2a thiols ( $0.4 \mathrm{mmol}, 2.0$ equiv), $\mathrm{Ph}_{3} \mathrm{P}$ ( $0.4 \mathrm{mmol}, 2.0$ equiv), TFA (10 mol \%), DMA $(2.0 \mathrm{~mL}), \mathrm{rt}, 5 \mathrm{~W}$ blue LEDs, $24-36 \mathrm{~h}$. 
aromatic ring of the aldehyde moiety bearing both electrondonating (e.g., $-\mathrm{MeO}$ and $-\mathrm{NHAc}$ ) and electron-withdrawing (e.g., - $\mathrm{F},-\mathrm{Br},-\mathrm{CN}$, -COOMe, and $-\mathrm{CF}_{3}$ ) substituents were examined $(3 \mathbf{k}-3 \mathbf{r})$, the reaction proceeded well in $49-81 \%$ yield. The position of the substituents on the aromatic ring seemed to have no obvious effect on the efficiency $(3 s-3 v)$. In addition, thiophene-aldehyde-derived imine was a good coupling partner with $48 \%$ yield $(3 \mathbf{x})$. Finally, we focused our attention on exploring the influence of the $N$-aryl moiety of imine, and the reaction worked well with a number of substituents on the aniline portion. Both electron-rich (-OMe and $-\mathrm{NHAc}$ ) and electron-poor $(-\mathrm{F}$ and $-\mathrm{Cl}$ ) groups were well tolerated by reverse polarity $(3 y-3 c c)$. It was noteworthy that an iodo group sensitive to a transition metal could tolerate the conditions well, and this offered great possibilities for further functionalization by transition metal-catalyzed coupling (3dd).

For this desulfurizing benylation protocol, ${ }^{19}$ we further explored the feasibility of large-scale synthesis by utilizing continuous-flow microtubing reactors (Scheme 4). It could

Scheme 4. A Large-Scale Synthesis Utilizing ContinuousFlow Microtubing Reactors

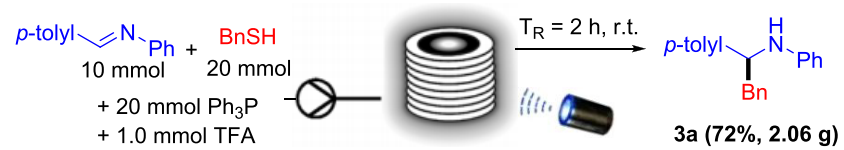

In batch: $2 \mathrm{~mol} \% \operatorname{Ir}\left[\mathrm{dF}\left(\mathrm{CF}_{3}\right) \mathrm{ppy}\right]_{2}(\mathrm{dtbbpy}) \mathrm{PF}_{6}, 48 \mathrm{~h}$, slow and expensive In flow: $0.1 \mathrm{~mol} \% \operatorname{Ir}\left[\mathrm{dF}_{(}\left(\mathrm{CF}_{3}\right) \mathrm{ppy}_{2}(\mathrm{dtbbpy}) \mathrm{PF}_{6}, 2 \mathrm{~h}\right.$, faster and cheaper

serve as an enabling technology to decrease the reaction times of photocatalytic transformations from $48 \mathrm{~h}$ in batch to $2 \mathrm{~h}$ in flow. Notably, the requirement of a photocatalyst $\{\operatorname{Ir}[\mathrm{dF}$ $\left(\mathrm{CF}_{3}\right)$ ppy $]_{2}$ (dtbbpy) $\left.\mathrm{PF}_{6}\right\}$ was dramatically decreased in continuous-flow photomicroreactors from 2 to $0.1 \mathrm{~mol} \%$ compared to that of the batch synthesis, highlighting the superiority of flow chemistry in photocatalytic synthesis. ${ }^{20}$

To gain insight into the mechanism of this desulfurizing benzylation reaction, a series of control reactions were carried out as shown in Scheme 5 (or see the Supporting

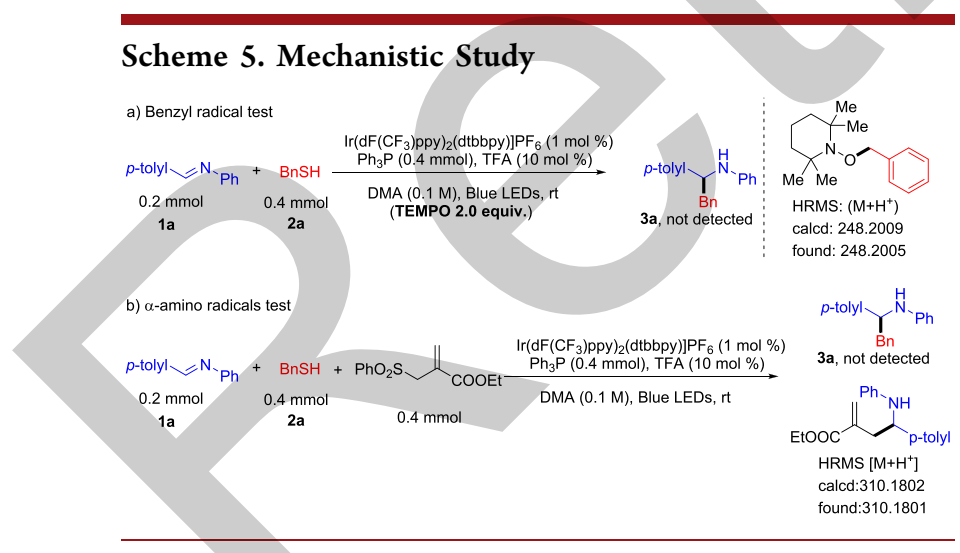

Information). The desired reaction could be prohibited completely with TEMPO added to the reaction system, and the TEMPO-trapped product could be detected by HRMS. In addition, the imine dimerization byproduct could be observed, indicating that the $\alpha$-amino alkyl radical might be involved under standard conditions. The corresponding Stern-Volmer experiments further revealed that photoexcited $*\left[\operatorname{Ir}\left(\mathrm{dF}\left(\mathrm{CF}_{3}\right)\right.\right.$ ppy $\left.)_{2}(\mathrm{dtbbpy})\right] \mathrm{PF}_{6}$ was quenched well by benzyl thiol. The quantum yield ( $\Phi=0.41$ ) was measured to certify that the photocatalyst could be regenerated during the reaction.

On the basis of the literature and our experiments, a possible mechanism is proposed in Scheme 6. This desulfurizing

Scheme 6. Proposed Possible Mechanism

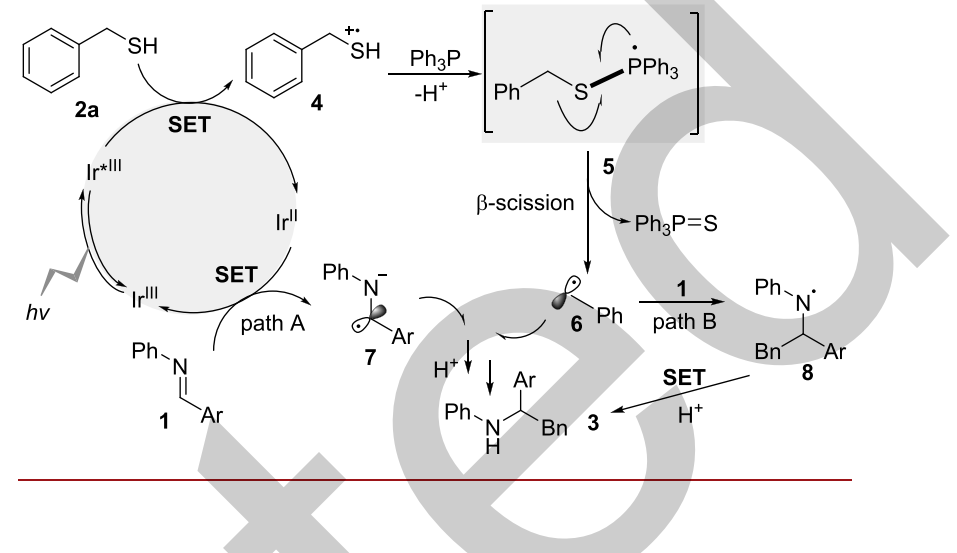

benzylation of imines with thiols is achieved by the combination of the photocatalytic reverse polarity of imine with the phosphoranyl-assisted desulfurization process. Photoexcited $*\left[\operatorname{Ir}\left(\mathrm{dF}\left(\mathrm{CF}_{3}\right) \text { ppy }\right)_{2}(\mathrm{dtbbpy})\right] \mathrm{PF}_{6}\left[E_{1 / 2}^{\text {red }}\left(* \mathrm{Ir}^{\mathrm{III}} / \mathrm{Ir}^{\mathrm{II}}\right)=\right.$ $1.21 \mathrm{~V}$ vs SCE; $\tau=2.3 \mu \mathrm{s}]^{21}$ can undergo single-electron transfer (SET) oxidation with thiol to generate thiyl radical cation $4 .{ }^{11 \mathrm{~b}}$ This radical cation could react with $\mathrm{Ph}_{3} \mathrm{P}$ to deliver a phosphoranyl radical for achieving the desulfurization process, ${ }^{17 \mathrm{~b}}$ giving rise to a benzyl radical 6 . The SET reduction of the imine with the reductive $\operatorname{Ir}^{\mathrm{II}}$ species furnished $\alpha$-amino radical anion 7 , followed by radical-radical $\mathrm{C}-\mathrm{C}$ coupling with a benzyl radical 6 to give the final $\alpha$-benzyl amine product 3 (path A). Alternatively, the pathway of addition of the radical to imines cannot be ruled out completely at this stage (path B).

In conclusion, the first desulfurizing benzylation reaction of imine with benzyl thiols has been achieved by visible light photoredox catalysis. The phosphoranyl-assisted desulfurization process as the key step furnishes benzyl radicals, which are utilized for subsequent $\mathrm{C}-\mathrm{C}$ coupling with $\alpha$-amino radical anions generated by photoinduced reductive SET umpolung of imine. The benzyl group can easily be installed to deliver a variety of $\alpha$-benzyl amine products under the mild conditions of our photoredox process.

\section{ASSOCIATED CONTENT \\ Supporting Information}

The Supporting Information is available free of charge at https://pubs.acs.org/doi/10.1021/acs.orglett.0c00065.

Experimental procedures and characterization data (PDF)

\section{AUTHOR INFORMATION}

\section{Corresponding Author}

Muliang Zhang - Department of Chemistry, National University of Singapore, Republic of Singapore 117543; ○ orcid.org/0000-0002-0415-9444; Email: CHMMZ@ nus.edu.sg

\section{Author}

Jinghui Zhang - Beijing Key Laboratory for Science and Application of Functional Molecular and Crystalline Materials, 
Department of Chemistry, University of Science and Technology Beijing, Beijing 100083, China

Complete contact information is available at:

https://pubs.acs.org/10.1021/acs.orglett.0c00065

\section{Notes}

The authors declare no competing financial interest.

\section{ACKNOWLEDGMENTS}

The authors gratefully acknowledge the financial support provided by the Ministry of Education (MOE) of Singapore (MOE2017-T2-2-081) and the National Natural Science Foundation of China (21671017 and 31371203).

\section{REFERENCES}

(1) Patai, S., Ed. The chemistry of the thiol group; Wiley: London, 1974

(2) (a) Dénès, F.; Pichowicz, M.; Povie, G.; Renaud, P. Chem. Rev. 2014, 114, 2587. (b) Schoneich, C. Molecules 2019, 24, 4357.

(3) (a) Licht, S.; Gerfen, G. J.; Stubbe, J. Science 1996, 271, 477. (b) Kolberg, M.; Strand, K. R.; Graff, P.; Andersson, K. K. Biochim. Biophys. Acta, Proteins Proteomics 2004, 1699, 1.

(4) Torrents, E.; Aloy, P.; Gibert, I.; Rodríguez-Trelles, F. J. Mol. Evol. 2002, 55, 138.

(5) (a) Ye, J.-H.; Miao, M.; Huang, H.; Yan, S.-S.; Yin, Z.-B.; Zhou, W.-J.; Yu, D.-G. Angew. Chem., Int. Ed. 2017, 56, 15416. (b) Wang, H.; Lu, Q.; Qian, C.; Liu, C.; Liu, W.; Chen, K.; Lei, A. Angew. Chem., Int. Ed. 2016, 55, 1094. (c) Goddard-Borger, E. D.; Tropak, M. B.; Yonekawa, S.; Tysoe, C.; Mahuran, D. J.; Withers, S. G. J. Med. Chem. 2012, 55, 2737. (d) Teders, M.; Henkel, C.; Anhäuser, L.; StriethKalthoff, F.; Gómez-Suárez, A.; Kleinmans, R.; Kahnt, A.; Rentmeister, A.; Guldi, D.; Glorius, F. Nat. Chem. 2018, 10, 981. (e) Yuan, Y.; Chen, Y.; Tang, S.; Huang, Z.; Lei, A. Sci. Adv. 2018, 4, eaat5312.

(6) (a) Liu, Y.; Hou, W.; Sun, H.; Cui, C.; Zhang, L.; Jiang, Y.; Wu, Y.; Wang, Y.; Li, J.; Sumerlin, B. S.; Liu, Q.; Tan, W. Chem. Sci. 2017, 8, 6182. (b) Hoyle, C. E.; Bowman, C. N. Angew. Chem., Int. Ed. 2010, 49, 1540. (c) Lowe, A. B. Polym. Chem. 2014, 5, 4820. (d) Dondoni, A. Angew. Chem., Int. Ed. 2008, 47, 8995.

(7) (a) Wille, U. Chem. Rev. 2013, 113, 813. (b) Zhu, Y.; Lowe, A. B.; Roth, P. J. Polymer 2014, 55, 4425.

(8) Crich, D.; Quintero, L. Chem. Rev. 1989, 89, 1413.

(9) (a) Majumdar, K. C.; Debnath, P. Tetrahedron 2008, 64, 9799.

(b) Zhang, G.; Liu, C.; Yi, H.; Meng, Q.; Bian, C.; Chen, H.; Jian, J. X.; Wu, L. Z.; Lei, A. J. Am. Chem. Soc. 2015, 137, 9273.

(10) Barton, D. H. R.; Crich, D. Tetrahedron Lett. 1985, 26, 757.

(11) (a) Zhu, F.; Miller, E.; Zhang, S.-q.; Yi, D.; O’Neill, S.; Hong, X.; Walczak, M. A. J. Am. Chem. Soc. 2018, 140, 18140. (b) Oderinde, M. S.; Frenette, M.; Robbins, D. W.; Aquila, B.; Johannes, J. W. J. Am. Chem. Soc. 2016, 138, 1760. (c) Santandrea, J.; Minozzi, C.; Cruché, C.; Collins, S. K. Angew. Chem., Int. Ed. 2017, 56, 12255. (d) Huang, P.; Wang, P.; Tang, S.; Fu, Z.; Lei, A. Angew. Chem., Int. Ed. 2018, 57, 8115. (e) Vara, B. A.; Li, X.; Berritt, S.; Walters, C. R.; Petersson, E. J.; Molander, G. A. Chem. Sci. 2018, 9, 336.

(12) Capaldo, L.; Ravelli, D. Eur. J. Org. Chem. 2017, 2017, 2056.

(13) For recent representative examples of the inert bond of substrates by thiols, see: (a) Ren, S.-C.; Zhang, F.-L.; Xu, A.-Q.; Yang, Y.; Zheng, M.; Zhou, X.; Fu, Y.; Wang, Y.-F. Nat. Commun. 2019, 10, 1934. (b) Kawamoto, T.; Geib, S. J.; Curran, D. P. J. Am. Chem. Soc. 2015, 137, 8617. (c) Ren, S.-C.; Zhang, F.-L.; Qi, J.; Huang, Y.-S.; Xu, A.-Q.; Yan, H.-Y.; Wang, Y.-F. J. Am. Chem. Soc. 2017, 139, 6050. (d) Meng, Q.-Y.; Schirmer, T. E.; Berger, A. L.; Donabauer, K.; König, B. J. Am. Chem. Soc. 2019, 141, 11393. (e) Cuthbertson, J. D.; MacMillan, D. W. Nature 2015, 519, 74. (f) Yu, W.-L.; Luo, Y.-C.; Yan, L.; Liu, D.; Wang, Z.-Y.; Xu, P.-F. Angew. Chem., Int. Ed. 2019, $58,10941$.
(14) For recent representative examples of radicals trapped by thiols, see: (a) Soulard, V.; Villa, G.; Vollmar, D. P.; Renaud, P. J. Am. Chem. Soc. 2018, 140, 155. (b) Loh, Y. Y.; Nagao, K.; Hoover, A. J.; Hesk, D.; Rivera, N. R.; Colletti, S. L.; Davies, I. W.; MacMillan, D. W. C. Science 2017, 358, 1182. (c) Shin, N. Y.; Ryss, J. M.; Zhang, X.; Miller, S. J.; Knowles, R. R. Science 2019, 366, 364. (d) Wu, F.; Wang, L.; Chen, J.; Nicewicz, D. A.; Huang, Y. Angew. Chem., Int. Ed. 2018, 57, 2174. (e) Zhang, M.; Yuan, X.-A.; Zhu, C.; Xie, J. Angew. Chem., Int. Ed. 2019, 58, 312.

(15) (a) Jia, K.; Pan, Y.; Chen, Y. Angew. Chem., Int. Ed. 2017, 56, 2478. (b) Basch, C. H.; Liao, J.; Xu, J.; Piane, J. J.; Watson, M. P. J. Am. Chem. Soc. 2017, 139, 5313.

(16) (a) Yus, M.; Martínez, P.; Guijarro, D. Synth. Commun. 2003, 33, 2365. (b) Rentner, J.; Kljajic, M.; Offner, L.; Breinbauer, R. Tetrahedron 2014, 70, 8983.

(17) (a) Benati, L.; Leardini, R.; Minozzi, M.; Nanni, D.; Scialpi, R.; Spagnolo, P.; Strazzari, S.; Zanardi, G. Angew. Chem., Int. Ed. 2004, 43, 3598. (b) Zhang, L.; Si, X.; Yang, Y.; Witzel, S.; Sekine, K.; Rudolph, M.; Rominger, F.; Hashmi, A. S. K. ACS Catal. 2019, 9, 6118.

(18) For recent representative examples of imines, see: (a) Lee, K. N.; Lei, Z.; Ngai, M.-Y. J. Am. Chem. Soc. 2017, 139, 5003. (b) Fan, X.; Gong, X.; Ma, M.; Wang, R.; Walsh, P. J. Nat. Commun. 2018, 9, 4936. (c) Uraguchi, D.; Kinoshita, N.; Kizu, T.; Ooi, T. J. Am. Chem. Soc. 2015, 137, 13768. (d) Fava, E.; Millet, A.; Nakajima, M.; Loescher, S.; Rueping, M. Angew. Chem., Int. Ed. 2016, 55, 6776. (e) Qi, L.; Chen, Y. Angew. Chem., Int. Ed. 2016, 55, 13312. (f) Han, B.; Li, Y.; Yu, Y.; Gong, L. Nat. Commun. 2019, 10, 3804. (g) Hager, D.; MacMillan, D. W. J. Am. Chem. Soc. 2014, 136, 16986.

(19) (a) Kong, D.; Moon, P. J.; Bsharat, O.; Lundgren, R. J. Angew. Chem., Int. Ed. 2020, 59, 1313. (b) Moon, P. J.; Fahandej-Sadi, A.; Qian, W.; Lundgren, R. J. Angew. Chem., Int. Ed. 2018, 57, 4612.

(20) (a) Lima, F.; Kabeshov, M. A.; Tran, D. N.; Battilocchio, C.; Sedelmeier, J.; Sedelmeier, G.; Schenkel, B.; Ley, S. V. Angew. Chem., Int. Ed. 2016, 55, 14085. (b) Deng, H.-P.; Zhou, Q.; Wu, J. Angew. Chem., Int. Ed. 2018, 57, 12661.

(21) Prier, C. K.; Rankic, D. A.; MacMillan, D. W. C. Chem. Rev. 2013, 113, 5322 . 


\section{SUPPORTING INFORMATION}

\section{Harnessing Thiol as Benzyl Reagent for Photocatalytic Reductive Benzylation of Imines}

Jinghui Zhang ${ }^{[a]}$ and Muliang Zhang, ${ }^{[b] *}$

${ }^{a}$ Beijing Key Laboratory for Science and Application of Functional Molecular and Crystalline Materials, Department of Chemistry, University of Science and Technology Beijing, Beijing 100083, China

${ }^{b}$ Department of Chemistry, National University of Singapore, 3 Science Drive 3, 117543 (Republic of Singapore)

\section{Table of Contents}

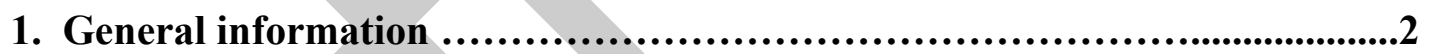

2. General procedure for desulfurizing cross-coupling .........................................3

3. Mechanistic Investigations........................................................18

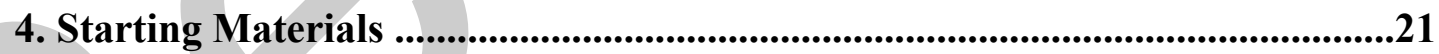

5. Copies of ${ }^{1} \mathrm{H}$ NMR, ${ }^{13} \mathrm{C}$ NMR Spectra..............................................21

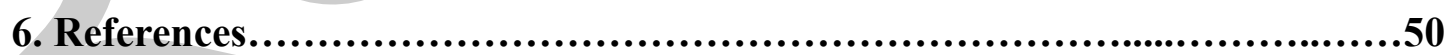




\section{General information}

All reactions were carried out under Ar atmosphere unless otherwise noted. All reagents and solvents were obtained from commercial suppliers and used without further purification. Reactions were monitored by TLC on silica gel plates $\left(\mathrm{GF}_{254}\right)$, and the analytical thin-layer chromatography (TLC) was performed on precoated, glass-backed silica gel plates. ${ }^{1} \mathrm{H}$ NMR, and ${ }^{13} \mathrm{C}$ NMR spectra were recorded on a Bruker AVANCE III-400 spectrometer at room temperature. Chemical shifts $(\delta)$ are reported in ppm downfield from tetramethylsilane. Abbreviations for signal couplings are: s, singlet; d, doublet; t, triplet; m, multiplet; broad (br). High resolution mass spectra were obtained using an Agilent 6210 Series TOF LC-MS equipped with electrospray ionization (ESI) probe operating in positive ion mode. HPLC analysis was performed on Shimadzu SPD-20A using Daicel Chiralpak AD Column. The $5 \mathrm{~W}$ blue LED lamps $\left(\lambda_{\max }=455 \mathrm{~nm}\right)$ and $18 \mathrm{~W}$ blue LED lamps were directly got from the supermarket.

\section{General procedure for desulfurizing cross-coupling}

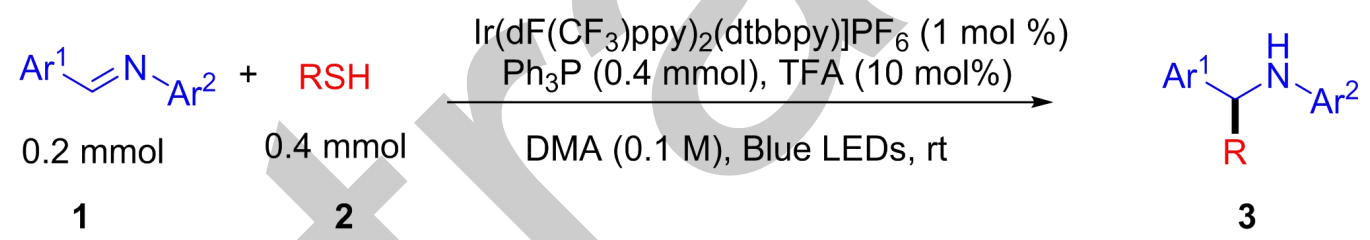

To a $10 \mathrm{~mL}$ Schlenk tube equipped with a magnetic stir bar was added imine $(0.2$ mmol, 1.0 equiv. $)$, photocatalyst $\operatorname{Ir}\left[\mathrm{dF}\left(\mathrm{CF}_{3}\right) \text { ppy }\right]_{2}(\mathrm{dtbbpy}) \mathrm{PF}_{6}(2.3 \mathrm{mg}, 1 \mathrm{~mol} \%)$ and $\mathrm{Ph}_{3} \mathrm{P}$ (104.8 mg, $0.4 \mathrm{mmol}, 2.0$ equiv.) and the tube was evacuated and backfilled with $\operatorname{Ar}$ (three times). Thiol (0.4 mmol, 2.0 equiv.) and the additive TFA (2.3 mg, $10 \mathrm{~mol} \%)$ in DMA $(2.0 \mathrm{~mL})$ were added by syringe under Ar. The tube was then sealed and was placed at a distance (app. $5 \mathrm{~cm}$ ) from $5 \mathrm{~W}$ blue LEDs lamp, and the mixture was stirred for $24-36 \mathrm{~h}$ at room temperature. After completion, the mixture was quenched with water and extracted with EtOAc $(3 \times 10 \mathrm{~mL})$. The combined organic layer was dried over anhydrous $\mathrm{Na}_{2} \mathrm{SO}_{4}$, then the solvent was removed under vacuo. The residue was purified with chromatography column on silica gel (gradient eluent of petroleum ether/EtOAc) to give the corresponding $\alpha$-benzyl amine products 3 . 


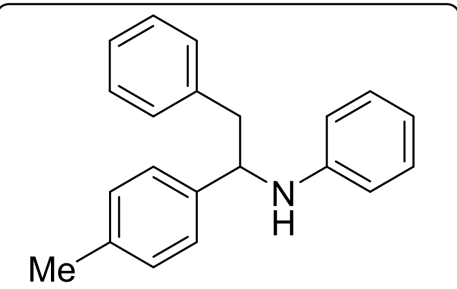

$N$-(2-phenyl-1-(p-tolyl)ethyl)aniline 3a

The reaction was carried out according to the general procedure on $0.2 \mathrm{mmol}$ scale (36 h). The residue was purified by flash column chromatography (petroleum ether: ethyl acetate, 100/1-50/1) to afford 3a, (80\%, $45.9 \mathrm{mg})$, a colorless sticky oil. ${ }^{1} \mathrm{H}$ NMR (400 MHz, Chloroform-d) $\delta 7.28-7.20(\mathrm{~m}, 5 \mathrm{H}), 7.15-7.08(\mathrm{~m}, 4 \mathrm{H}), 7.06-$ $7.01(\mathrm{~m}, 2 \mathrm{H}), 6.61(\mathrm{t}, J=7.3 \mathrm{~Hz}, 1 \mathrm{H}), 6.45(\mathrm{~d}, J=7.7 \mathrm{~Hz}, 2 \mathrm{H}), 4.55(\mathrm{dd}, J=8.3,5.7$ $\mathrm{Hz}, 1 \mathrm{H}), 3.11(\mathrm{dd}, J=14.0,5.7 \mathrm{~Hz}, 1 \mathrm{H}), 2.98(\mathrm{dd}, J=14.0,8.3 \mathrm{~Hz}, 1 \mathrm{H}), 2.32(\mathrm{~s}, 3 \mathrm{H})$. ${ }^{13} \mathrm{C}$ NMR (100 MHz, Chloroform- $d$ ) $\delta$ 147.4, 140.5, 137.9, 136.6, 129.3, 129.2, 129.0, 128.6, 126.7, 126.4, 117.4, 113.7, 59.0, 45.2, 21.2. HRMS (ESI) Calculated for $\mathrm{C}_{21} \mathrm{H}_{22} \mathrm{~N}^{+}\left([\mathrm{M}+\mathrm{H}]^{+}\right): 288.1747$, found: 288.1749 .

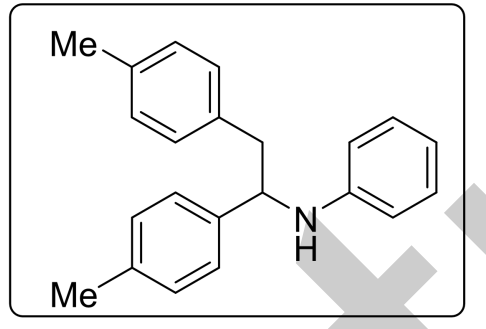

$N$-(1,2-di- $p$-tolylethyl)aniline $\mathbf{3 b}$

The reaction was carried out according to the general procedure on $0.2 \mathrm{mmol}$ scale $(36 \mathrm{~h})$. The residue was purified by flash column chromatography (petroleum ether: ethyl acetate, 100/1-50/1) to afford 3b, (74\%, $44.7 \mathrm{mg})$, a colorless sticky oil. ${ }^{1} \mathrm{H}$ NMR (400 MHz, Chloroform- $d$ ) $\delta 7.14(\mathrm{~d}, J=7.9 \mathrm{~Hz}, 2 \mathrm{H}), 7.05-6.89(\mathrm{~m}, 8 \mathrm{H}), 6.53$ $(\mathrm{t}, J=7.3 \mathrm{~Hz}, 1 \mathrm{H}), 6.37(\mathrm{~d}, J=7.8 \mathrm{~Hz}, 2 \mathrm{H}), 4.44(\mathrm{dd}, J=8.2,5.6 \mathrm{~Hz}, 1 \mathrm{H}), 3.01(\mathrm{dd}, J$ $=14.0,5.4 \mathrm{~Hz}, 1 \mathrm{H}), 2.85(\mathrm{dd}, J=14.0,8.4 \mathrm{~Hz}, 1 \mathrm{H}), 2.24(\mathrm{~s}, 3 \mathrm{H}), 2.23(\mathrm{~s}, 3 \mathrm{H}) .{ }^{13} \mathrm{C}$ NMR (100 MHz, Chloroform-d) $\delta$ 147.46, 140.61, 136.52, 136.21, 134.71, 129.29, $129.25,129.08,129.00,126.36,117.35,113.64,58.94,44.84,21.15,21.09$. HRMS (ESI) Calculated for $\mathrm{C}_{22} \mathrm{H}_{24} \mathrm{~N}^{+}\left([\mathrm{M}+\mathrm{H}]^{+}\right): 302.1903$, found: 302.1904 . 


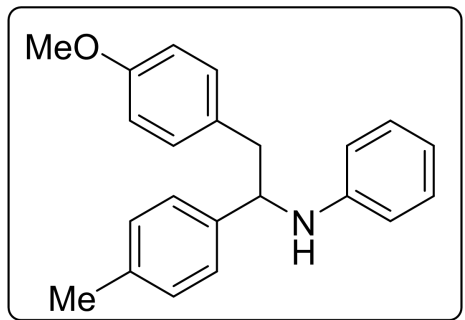

$N$-(2-(4-methoxyphenyl)-1-(p-tolyl)ethyl)aniline 3c

The reaction was carried out according to the general procedure on $0.2 \mathrm{mmol}$ scale (36 h). The residue was purified by flash column chromatography (petroleum ether: ethyl acetate, 100/1-60/1) to afford 3c, (56\%, $35.5 \mathrm{mg})$, a colorless sticky oil. ${ }^{1} \mathrm{H}$ NMR (400 MHz, Chloroform- $d$ ) $\delta 7.22(\mathrm{~d}, J=8.0 \mathrm{~Hz}, 2 \mathrm{H}), 7.13-7.02(\mathrm{~m}, 6 \mathrm{H}), 6.82$ (d, $J=8.6 \mathrm{~Hz}, 2 \mathrm{H}), 6.63$ (t, $J=7.3 \mathrm{~Hz}, 1 \mathrm{H}), 6.47(\mathrm{~d}, J=7.7 \mathrm{~Hz}, 2 \mathrm{H}), 4.62-4.37$ (m, 1H), 3.79 (s, 3H), $3.08(\mathrm{dd}, J=14.1,5.7 \mathrm{~Hz}, 1 \mathrm{H}), 2.94$ (dd, $J=14.1,8.1 \mathrm{~Hz}, 1 \mathrm{H})$, $2.34(\mathrm{~s}, 3 \mathrm{H}) .{ }^{13} \mathrm{C}$ NMR (100 MHz, Chloroform- $d$ ) $\delta 158.37,147.43,140.50,136.51$, $130.19,129.79,129.25,128.99,126.36,117.36,113.93,113.63,59.03,55.25,44.31$, 21.13. HRMS (ESI) Calculated for $\mathrm{C}_{22} \mathrm{H}_{24} \mathrm{NO}^{+}\left([\mathrm{M}+\mathrm{H}]^{+}\right): 318.1852$, found: 318.1855 .

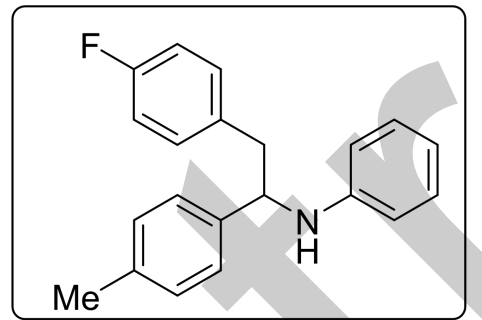

$N$-(2-(4-fluorophenyl)-1-(p-tolyl)ethyl)aniline 3d

The reaction was carried out according to the general procedure on $0.2 \mathrm{mmol}$ scale $(36 \mathrm{~h})$. The residue was purified by flash column chromatography (petroleum ether: ethyl acetate, 100/1-50/1) to afford 3d, (58\%, $35.4 \mathrm{mg})$, a colorless sticky oil. ${ }^{1} \mathrm{H}$ NMR (400 MHz, Chloroform- $d$ ) $\delta 7.22-7.01(\mathrm{~m}, 8 \mathrm{H}), 6.95(\mathrm{t}, J=8.7 \mathrm{~Hz}, 2 \mathrm{H}), 6.64$ (t, $J=7.3 \mathrm{~Hz}, 1 \mathrm{H}), 6.48(\mathrm{~d}, J=7.7 \mathrm{~Hz}, 2 \mathrm{H}), 4.64-4.38(\mathrm{~m}, 1 \mathrm{H}), 4.10$ (br s, 1H), 3.26 - 2.87 (m, 2H), $2.33(\mathrm{~s}, 3 \mathrm{H}) .{ }^{13} \mathrm{C}$ NMR (100 MHz, Chloroform- $\left.d\right) \delta 161.72(\mathrm{~d}, J=$ $244.7 \mathrm{~Hz}), 147.19,139.99,136.70,133.47,130.66$ (d, $J=7.9 \mathrm{~Hz}), 129.17$ (d, $J=23.2$ Hz), 126.36, 117.53, 115.38, 115.17, 113.59, 58.98, 44.16, 21.11. HRMS (ESI) Calculated for $\mathrm{C}_{21} \mathrm{H}_{21} \mathrm{FN}^{+}\left([\mathrm{M}+\mathrm{H}]^{+}\right)$: 306.1653 , found: 306.1655 . 


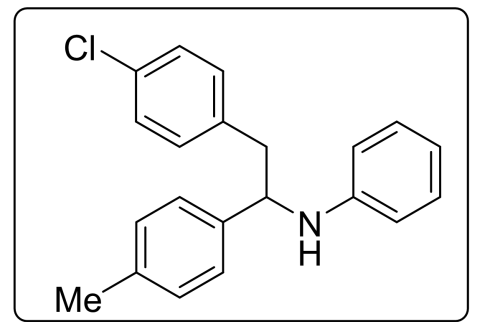

N-(2-(4-chlorophenyl)-1-(p-tolyl)ethyl)aniline 3e

The reaction was carried out according to the general procedure on $0.2 \mathrm{mmol}$ scale (36 h). The residue was purified by flash column chromatography (petroleum ether: ethyl acetate, 100/1-50/1) to afford 3e, $(67 \%, 43.0 \mathrm{mg})$, as a white solid. ${ }^{1} \mathrm{H}$ NMR $(400 \mathrm{MHz}$, Chloroform- $d$ ) $\delta 7.37-6.96(\mathrm{~m}, 10 \mathrm{H}), 6.65$ (t, $J=7.3 \mathrm{~Hz}, 1 \mathrm{H}), 6.49$ (d, $J$ $=8.5 \mathrm{~Hz}, 2 \mathrm{H}), 4.55(\mathrm{t}, J=6.9 \mathrm{~Hz}, 1 \mathrm{H}), 4.05(\mathrm{br} \mathrm{s}, 0 \mathrm{H}), 3.09-2.99(\mathrm{~m}, 2 \mathrm{H}), 2.33(\mathrm{~s}$, $3 \mathrm{H}) .{ }^{13} \mathrm{C}$ NMR (100 MHz, Chloroform- $d$ ) $\delta 147.11,139.85,136.77,136.27,132.46$, $130.59,129.33,129.09,128.58,126.37,117.59,113.60,58.81,44.26,21.12$. HRMS (ESI) Calculated for $\mathrm{C}_{21} \mathrm{H}_{21} \mathrm{ClN}^{+}\left([\mathrm{M}+\mathrm{H}]^{+}\right): 322.1357$, found: 322.1358 .
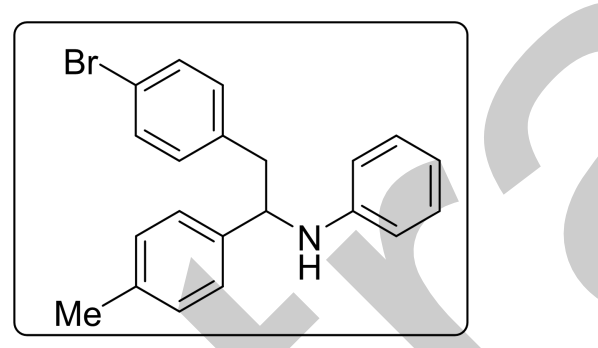

$N$-(2-(4-bromophenyl)-1-(p-tolyl)ethyl)aniline $3 \mathbf{f}$

The reaction was carried out according to the general procedure on $0.2 \mathrm{mmol}$ scale (36 h). The residue was purified by flash column chromatography (petroleum ether: ethyl acetate, 100/1-50/1) to afford $\mathbf{3 f},(68 \%, 49.6 \mathrm{mg})$, as a white solid. ${ }^{1} \mathrm{H}$ NMR (400 MHz, Chloroform- $d$ ) $\delta 7.39(\mathrm{~d}, J=8.3 \mathrm{~Hz}, 2 \mathrm{H}), 7.21-7.04(\mathrm{~m}, 6 \mathrm{H}), 6.97(\mathrm{~d}, J=$ $8.3 \mathrm{~Hz}, 2 \mathrm{H}), 6.65$ (t, $J=7.3 \mathrm{~Hz}, 1 \mathrm{H}), 6.49(\mathrm{~d}, J=8.6 \mathrm{~Hz}, 2 \mathrm{H}), 4.55$ (t, $J=6.9 \mathrm{~Hz}, 1 \mathrm{H})$, $3.14-2.91(\mathrm{~m}, 2 \mathrm{H}), 2.34(\mathrm{~s}, 3 \mathrm{H}) \cdot{ }^{13} \mathrm{C}$ NMR (100 MHz, Chloroform-d) $\delta$ 147.09, $139.82,136.79,131.53,130.99$, 129.34, 129.09, 126.38, 120.54, 117.61, 113.60, 58.74, 44.30, 21.13. HRMS (ESI) Calculated for $\mathrm{C}_{21} \mathrm{H}_{21} \mathrm{BrN}^{+}\left([\mathrm{M}+\mathrm{H}]^{+}\right)$: 366.0852, found: 366.0854 . 


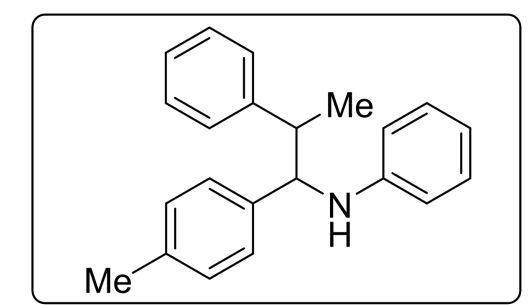

$N$-(2-phenyl-1-(p-tolyl)propyl)aniline $\mathbf{3 g}$

The reaction was carried out according to the general procedure on $0.2 \mathrm{mmol}$ scale (24 h). The residue was purified by flash column chromatography (petroleum ether: ethyl acetate, 100/1-50/1) to afford $\mathbf{3 g}$, (70\%, $42.1 \mathrm{mg})$, a colorless sticky oil, (1:1 dr). ${ }^{1} \mathrm{H}$ NMR (400 MHz, Chloroform- $d$ ) $\delta 7.56-6.91$ (m, 10H), 6.62-6.48 (m, 1H), 6.45 $(\mathrm{d}, J=8.5 \mathrm{~Hz}, 1 \mathrm{H}), 6.36(\mathrm{~d}, J=8.5 \mathrm{~Hz}, 1 \mathrm{H}), 4.50(\mathrm{~d}, J=5.4 \mathrm{~Hz}, 0.5 \mathrm{H}), 4.31(\mathrm{~d}, J=$ $8.3 \mathrm{~Hz}, 0.5 \mathrm{H}), 3.34-3.16(\mathrm{~m}, 0.5 \mathrm{H}), 3.03-2.93(\mathrm{~m}, 0.5 \mathrm{H}), 2.31(\mathrm{~s}, 1.5 \mathrm{H}), 2.27(\mathrm{~s}$, 1.5H), $1.31(\mathrm{~d}, J=7.2 \mathrm{~Hz}, 1.5 \mathrm{H}), 1.15(\mathrm{~d}, J=7.0 \mathrm{~Hz}, 1.5 \mathrm{H})$. HRMS (ESI) Calculated for $\mathrm{C}_{22} \mathrm{H}_{24} \mathrm{~N}^{+}\left([\mathrm{M}+\mathrm{H}]^{+}\right)$: 302.1903, found: 302.1904 .

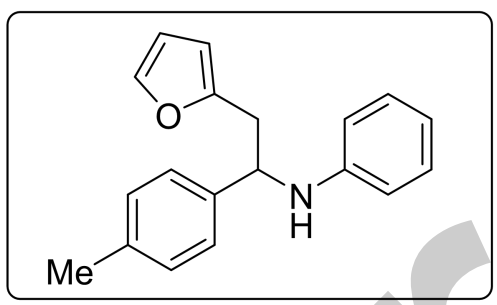

$N$-(2-(furan-2-yl)-1-(p-tolyl)ethyl)aniline $3 \mathbf{h}$

The reaction was carried out according to the general procedure on $0.2 \mathrm{mmol}$ scale (24 h). The residue was purified by flash column chromatography (petroleum ether: ethyl acetate, 100/1-60/1) to afford $\mathbf{3 h},(77 \%, 42.8 \mathrm{mg})$, a colorless sticky oil. ${ }^{1} \mathrm{H}$ NMR (400 MHz, Chloroform- $d$ ) $\delta 7.50-7.01(\mathrm{~m}, 6 \mathrm{H}), 6.62(\mathrm{t}, J=7.3 \mathrm{~Hz}, 1 \mathrm{H}), 6.48$ $(\mathrm{d}, J=7.7 \mathrm{~Hz}, 2 \mathrm{H}), 6.29-6.14(\mathrm{~m}, 1 \mathrm{H}), 6.01(\mathrm{~d}, J=3.1 \mathrm{~Hz}, 1 \mathrm{H}), 4.60(\mathrm{~d}, J=5.2 \mathrm{~Hz}$, 1H), $3.21-2.76(\mathrm{~m}, 2 \mathrm{H}), 2.31$ (s, 3H). ${ }^{13} \mathrm{C}$ NMR (100 MHz, Chloroform- $d$ ) $\delta$ 152.28, $147.40,141.82,140.20,136.78,129.41,129.09,126.18,117.50,113.65,110.37$, 107.47, 57.25, 37.45, 21.19. HRMS (ESI) Calculated for $\mathrm{C}_{19} \mathrm{H}_{20} \mathrm{NO}^{+}\left([\mathrm{M}+\mathrm{H}]^{+}\right)$: 278.1539, found: 278.1541 . 


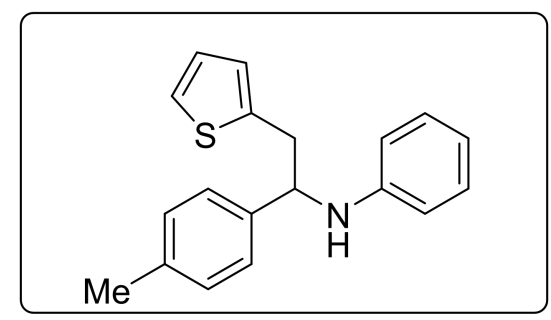

$N$-(2-(thiophen-2-yl)-1-(p-tolyl)ethyl)aniline $\mathbf{3 i}$

The reaction was carried out according to the general procedure on $0.2 \mathrm{mmol}$ scale (36 h). The residue was purified by flash column chromatography (petroleum ether: ethyl acetate, 100/1-50/1) to afford 3i, $(83 \%, 48.6 \mathrm{mg})$, a colorless sticky oil. ${ }^{1} \mathrm{H}$ NMR $(400 \mathrm{MHz}$, Chloroform- $d) \delta 7.29-6.98(\mathrm{~m}, 6 \mathrm{H}), 6.92-6.85(\mathrm{~m}, 1 \mathrm{H}), 6.77(\mathrm{~d}, J=4.3$ $\mathrm{Hz}, 1 \mathrm{H}), 6.63(\mathrm{t}, J=7.8 \mathrm{~Hz}, 1 \mathrm{H}), 6.53-6.44(\mathrm{~m}, 2 \mathrm{H}), 4.55(\mathrm{dd}, J=8.0,5.4 \mathrm{~Hz}, 1 \mathrm{H})$, $3.44-3.07(\mathrm{~m}, 2 \mathrm{H}), 2.31(\mathrm{~s}, 3 \mathrm{H}) .{ }^{13} \mathrm{C}$ NMR (100 MHz, Chloroform- $d$ ) $\delta$ 147.28, $140.25,139.94,136.91,129.42,129.13,126.87,126.41,126.21,124.44,117.65$, 113.73, 59.14, 39.06, 21.21. HRMS (ESI) Calculated for $\mathrm{C}_{19} \mathrm{H}_{20} \mathrm{NS}^{+}\left([\mathrm{M}+\mathrm{H}]^{+}\right)$: 294.1311, found: 294.1312 .<smiles>c1ccc(CC(Nc2ccccc2)c2ccccc2)cc1</smiles>

$N$-(1,2-diphenylethyl)aniline $\mathbf{3 k}$

The reaction was carried out according to the general procedure on $0.2 \mathrm{mmol}$ scale $(24 \mathrm{~h})$. The residue was purified by flash column chromatography (petroleum ether: ethyl acetate, 100/1-50/1) to afford 3k, (81\%, $44.2 \mathrm{mg})$, a colorless sticky oil. ${ }^{1} \mathrm{H}$ NMR (400 MHz, Chloroform-d) $\delta 7.35$ - $7.17(\mathrm{~m}, 8 \mathrm{H}), 7.12-7.10(\mathrm{~m}, 2 \mathrm{H}), 7.06-$ $7.02(\mathrm{~m}, 2 \mathrm{H}), 6.61(\mathrm{t}, J=7.8 \mathrm{~Hz}, 1 \mathrm{H}), 6.45(\mathrm{~d}, J=7.6 \mathrm{~Hz}, 2 \mathrm{H}), 4.58(\mathrm{dd}, J=8.2,5.7$ $\mathrm{Hz}, 1 \mathrm{H}), 3.13(\mathrm{dd}, J=14.0,5.7 \mathrm{~Hz}, 1 \mathrm{H}), 3.00(\mathrm{dd}, J=14.0,8.2 \mathrm{~Hz}, 1 \mathrm{H}) .{ }^{13} \mathrm{C}$ NMR (100 MHz, Chloroform-d) $\delta 147.3,143.5,137.7,129.2,129.1,128.6,128.6,127.1$, 126.8, 126.5, 117.5, 113.7, 59.3, 45.2. HRMS (ESI) Calculated for $\mathrm{C}_{20} \mathrm{H}_{20} \mathrm{~N}^{+}$ $\left([\mathrm{M}+\mathrm{H}]^{+}\right): 274.1590$, found: 274.1593 . 


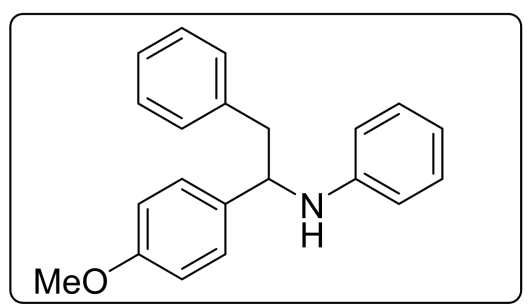

$\mathrm{N}$-(1-(4-methoxyphenyl)-2-phenylethyl)aniline 3I

The reaction was carried out according to the general procedure on $0.2 \mathrm{mmol}$ scale (24 h). The residue was purified by flash column chromatography (petroleum ether: ethyl acetate, 100/1-50/1) to afford 31, (60\%, $36.4 \mathrm{mg})$, as a white solid. ${ }^{1} \mathrm{H}$ NMR (400 MHz, Chloroform- $d$ ) $\delta 7.28-7.16(\mathrm{~m}, 5 \mathrm{H}), 7.12-6.99(\mathrm{~m}, 4 \mathrm{H}), 6.82(\mathrm{~d}, J=8.6 \mathrm{~Hz}$, $2 \mathrm{H}), 6.61(\mathrm{t}, J=7.3 \mathrm{~Hz}, 1 \mathrm{H}), 6.45(\mathrm{~d}, J=8.5 \mathrm{~Hz}, 2 \mathrm{H}), 4.53(\mathrm{dd}, J=5.9,7.9 \mathrm{~Hz}, 1 \mathrm{H})$, $3.76(\mathrm{~s}, 3 \mathrm{H}), 3.08(\mathrm{dd}, J=13.9,5.9 \mathrm{~Hz}, 1 \mathrm{H}), 2.99(\mathrm{dd}, J=13.9,7.9 \mathrm{~Hz}, 1 \mathrm{H}) .{ }^{13} \mathrm{C}$ NMR (100 MHz, Chloroform-d) $\delta 158.6,147.4,137.8,135.4,129.3,129.1,128.6$, 127.5, 126.7, 117.5, 114.0, 113.7, 58.7, 55.3, 45.3. HRMS (ESI) Calculated for $\mathrm{C}_{21} \mathrm{H}_{22} \mathrm{NO}^{+}\left([\mathrm{M}+\mathrm{H}]^{+}\right): 304.1696$, found: 304.1697 .

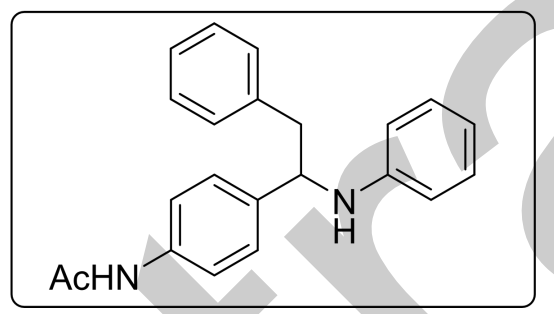

$\mathrm{N}$-(4-(2-phenyl-1-(phenylamino)ethyl)phenyl)acetamide 3m

The reaction was carried out according to the general procedure on $0.2 \mathrm{mmol}$ scale (24 h). The residue was purified by flash column chromatography (petroleum ether: ethyl acetate, 100/1-40/1) to afford $\mathbf{3 m},(55 \%, 36.3 \mathrm{mg})$, as a white solid. ${ }^{1} \mathrm{H}$ NMR (400 MHz, Chloroform-d) $\delta 7.42(\mathrm{~d}, J=8.5 \mathrm{~Hz}, 3 \mathrm{H}), 7.30-7.16(\mathrm{~m}, 5 \mathrm{H}), 7.13-6.99$ (m, 4H), 6.61 (t, $J=7.3 \mathrm{~Hz}, 1 \mathrm{H}), 6.43$ (d, $J=8.5 \mathrm{~Hz}, 2 \mathrm{H}), 4.54(\mathrm{dd}, J=8.0,5.8 \mathrm{~Hz}$, $1 \mathrm{H}), 3.08(\mathrm{dd}, J=13.9,5.8 \mathrm{~Hz}, 1 \mathrm{H}), 2.98(\mathrm{dd}, J=13.9,8.0 \mathrm{~Hz}, 1 \mathrm{H}), 2.12(\mathrm{~s}, 3 \mathrm{H}) .{ }^{13} \mathrm{C}$ NMR (100 MHz, Chloroform-d) $\delta$ 168.4, 147.2, 139.4, 137.6, 136.8, 129.2, 129.0, 128.6, 127.1, 126.8, 120.1, 117.6, 113.7, 58.8, 45.1, 24.6. HRMS (ESI) Calculated for $\mathrm{C}_{22} \mathrm{H}_{23} \mathrm{~N}_{2} \mathrm{O}^{+}\left([\mathrm{M}+\mathrm{H}]^{+}\right): 331.1805$, found: 331.1807 . 


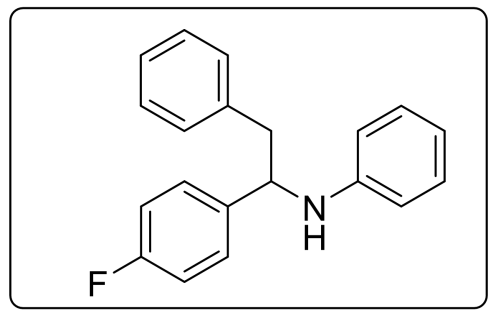

$N$-(1-(4-fluorophenyl)-2-phenylethyl)aniline 3n

The reaction was carried out according to the general procedure on $0.2 \mathrm{mmol}$ scale (24 h). The residue was purified by flash column chromatography (petroleum ether: ethyl acetate, 100/1-50/1) to afford $3 \mathbf{n},(68 \%, 39.6 \mathrm{mg})$, a colorless sticky oil. ${ }^{1} \mathrm{H}$ NMR (400 MHz, Chloroform- $d$ ) $\delta 7.29-7.23(\mathrm{~m}, 5 \mathrm{H}), 7.11-6.90(\mathrm{~m}, 6 \mathrm{H}), 6.64(\mathrm{t}, J$ $=7.3 \mathrm{~Hz}, 1 \mathrm{H}), 6.43(\mathrm{~d}, J=7.7 \mathrm{~Hz}, 2 \mathrm{H}), 4.55(\mathrm{dd}, J=6.3,7.9 \mathrm{~Hz}, 1 \mathrm{H}), 3.08(\mathrm{dd}, J=$ 13.9, $6.3 \mathrm{~Hz}, 1 \mathrm{H}), 3.01$ (dd, $J=13.8,7.9 \mathrm{~Hz}, 1 \mathrm{H}) .{ }^{13} \mathrm{C}$ NMR (100 MHz, Chloroform-d) $\delta 161.9(\mathrm{~d}, J=244.7 \mathrm{~Hz}), 147.1,139.0(\mathrm{~d}, J=3.1 \mathrm{~Hz}), 137.4,129.2,129.1,128.6$, $128.0(\mathrm{~d}, J=8.0 \mathrm{~Hz}), 126.8,117.7,115.4(\mathrm{~d}, J=21.4 \mathrm{~Hz}), 113.7,58.7,45.3$. HRMS (ESI) Calculated for $\mathrm{C}_{20} \mathrm{H}_{19} \mathrm{FN}^{+}\left([\mathrm{M}+\mathrm{H}]^{+}\right)$: 292.1496, found: 292.1499 .

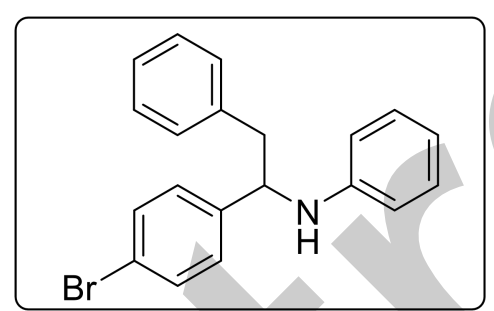

$\mathrm{N}$-(1-(4-bromophenyl)-2-phenylethyl)aniline 30

The reaction was carried out according to the general procedure on $0.2 \mathrm{mmol}$ scale $(24 \mathrm{~h})$. The residue was purified by flash column chromatography (petroleum ether: ethyl acetate, 100/1-50/1) to afford 3o, (65\%, $45.6 \mathrm{mg})$, as a white solid. ${ }^{1} \mathrm{H}$ NMR (400 MHz, Chloroform- $d$ ) $\delta 7.41(\mathrm{~d}, J=8.4 \mathrm{~Hz}, 2 \mathrm{H}), 7.32-7.15(\mathrm{~m}, 5 \mathrm{H}), 7.13-6.98$ (m, 4H), 6.64 (t, $J=7.3 \mathrm{~Hz}, 1 \mathrm{H}), 6.42$ (d, $J=9.5 \mathrm{~Hz}, 2 \mathrm{H}), 4.53(\mathrm{dd}, J=8.0,5.9 \mathrm{~Hz}$, $1 \mathrm{H}), 3.08(\mathrm{dd}, J=13.9,5.9 \mathrm{~Hz}, 1 \mathrm{H}), 2.99(\mathrm{dd}, J=13.9,8.0 \mathrm{~Hz}, 1 \mathrm{H}) .{ }^{13} \mathrm{C}$ NMR $(100$ MHz, Chloroform-d) $\delta 146.9,142.5,137.2,131.7,129.2,129.1,128.7,128.3,126.9$, 120.7, 117.8, 113.7, 58.8, 45.1. HRMS (ESI) Calculated for $\mathrm{C}_{20} \mathrm{H}_{19} \mathrm{BrN}^{+}\left([\mathrm{M}+\mathrm{H}]^{+}\right)$: 352.0695, found: 352.0698 . 


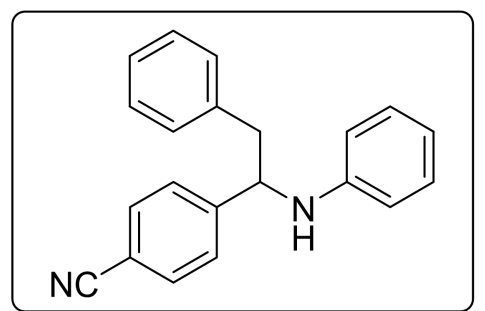

4-(2-phenyl-1-(phenylamino)ethyl)benzonitrile 3p

The reaction was carried out according to the general procedure on $0.2 \mathrm{mmol}$ scale (24 h). The residue was purified by flash column chromatography (petroleum ether: ethyl acetate, 100/1-40/1) to afford $\mathbf{3 p},(61 \%, 36.4 \mathrm{mg})$, a colorless sticky oil. ${ }^{1} \mathrm{H}$ NMR (400 MHz, Chloroform-d) $\delta 7.57$ (d, $J=8.2 \mathrm{~Hz}, 2 \mathrm{H}), 7.39$ (d, $J=8.2 \mathrm{~Hz}, 2 \mathrm{H}$ ), $7.29-7.22(\mathrm{~m}, 3 \mathrm{H}), 7.11-6.98(\mathrm{~m}, 4 \mathrm{H}), 6.66(\mathrm{t}, J=7.3 \mathrm{~Hz}, 1 \mathrm{H}), 6.39$ (d, $J=7.8 \mathrm{~Hz}$, 2H), $4.61(\mathrm{dd}, J=7.9,6.1 \mathrm{~Hz}, 1 \mathrm{H}), 3.08(\mathrm{dd}, J=13.9,6.1 \mathrm{~Hz}, 1 \mathrm{H}), 3.02(\mathrm{dd}, J=13.8$, $7.9 \mathrm{~Hz}, 1 \mathrm{H}) .{ }^{13} \mathrm{C}$ NMR $(100 \mathrm{MHz}$, Chloroform- $d$ ) $\delta 149.2,146.6,136.6,132.5,129.2$, 129.2, 128.8, 127.3, 127.2, 118.9, 118.2, 113.6, 111.0, 59.1, 44.8. HRMS (ESI) Calculated for $\mathrm{C}_{21} \mathrm{H}_{19} \mathrm{~N}_{2}{ }^{+}\left([\mathrm{M}+\mathrm{H}]^{+}\right)$: 299.1543, found: 299.1545 .

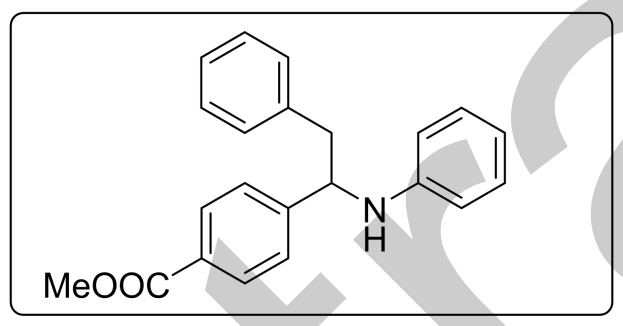

methyl 4-(2-phenyl-1-(phenylamino)ethyl)benzoate 3q

The reaction was carried out according to the general procedure on $0.2 \mathrm{mmol}$ scale (36 h). The residue was purified by flash column chromatography (petroleum ether: ethyl acetate, 100/1-30/1) to afford 3q, (67\%, $44.4 \mathrm{mg})$, as a white solid. ${ }^{1} \mathrm{H}$ NMR (400 MHz, Chloroform-d) $\delta 7.97$ (d, $J=8.3 \mathrm{~Hz}, 2 \mathrm{H}), 7.37$ (d, $J=8.2 \mathrm{~Hz}, 2 \mathrm{H}), 7.30$ $7.19(\mathrm{~m}, 3 \mathrm{H}), 7.14-6.97$ (m, 4H), 6.63 (t, $J=7.3 \mathrm{~Hz}, 1 \mathrm{H}), 6.42$ (d, $J=8.5 \mathrm{~Hz}, 2 \mathrm{H})$, $4.62(\mathrm{dd}, J=8.0,5.9 \mathrm{~Hz}, 1 \mathrm{H}), 3.89$ (s, 3H), 3.11 (dd, $J=13.9,5.9 \mathrm{~Hz}, 1 \mathrm{H}), 3.02(\mathrm{dd}$, $J=13.9,8.0 \mathrm{~Hz}, 1 \mathrm{H}) .{ }^{13} \mathrm{C} \mathrm{NMR}(100 \mathrm{MHz}$, Chloroform- $d) \delta 167.0,148.9,146.9$, 137.1, 130.0, 129.2, 129.1, 128.7, 127.0, 126.6, 117.8, 113.7, 59.2, 52.1, 44.9. HRMS (ESI) Calculated for $\mathrm{C}_{22} \mathrm{H}_{22} \mathrm{NO}_{2}{ }^{+}\left([\mathrm{M}+\mathrm{H}]^{+}\right): 332.1645$, found: 332.1646 . 


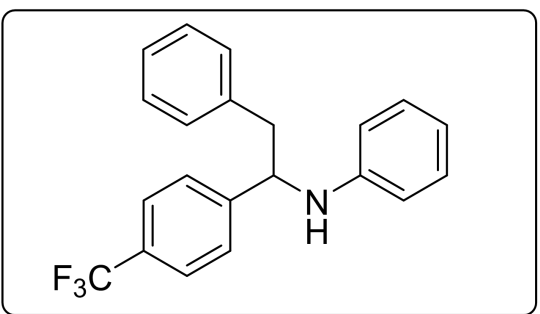

$N$-(2-phenyl-1-(4-(trifluoromethyl)phenyl)ethyl)aniline $3 r$

The reaction was carried out according to the general procedure on $0.2 \mathrm{mmol}$ scale (36 h). The residue was purified by flash column chromatography (petroleum ether: ethyl acetate, 100/1-50/1) to afford 3r, (49\%, $32.4 \mathrm{mg})$, a colorless sticky oil. ${ }^{1} \mathrm{H}$ NMR (400 MHz, Chloroform-d) $\delta 7.55$ (d, $J=8.1 \mathrm{~Hz}, 2 \mathrm{H}), 7.42$ (d, $J=8.2 \mathrm{~Hz}, 2 \mathrm{H})$, $7.32-7.21(\mathrm{~m}, 3 \mathrm{H}), 7.13-7.01(\mathrm{~m}, 4 \mathrm{H}), 6.70-6.60(\mathrm{~m}, 1 \mathrm{H}), 6.41(\mathrm{~d}, J=8.7 \mathrm{~Hz}$, 2H), $4.63(\mathrm{dd}, J=8.2,5.8 \mathrm{~Hz}, 1 \mathrm{H}), 3.12(\mathrm{dd}, J=14.0,5.8 \mathrm{~Hz}, 1 \mathrm{H}), 3.01(\mathrm{dd}, J=14.0$, $8.2 \mathrm{~Hz}, 1 \mathrm{H}) .{ }^{13} \mathrm{C}$ NMR (100 MHz, Chloroform- $d$ ) $\delta 147.7,146.8,137.0,129.4(\mathrm{q}, J=$ $32.1 \mathrm{~Hz}), 129.2,129.1,128.7,127.0,126.8,125.6$ (q, $J=3.7 \mathrm{~Hz}), 124.2$ (q, $J=270.2$ $\mathrm{Hz})$, 118.0, 113.7, 59.0, 45.0. HRMS (ESI) Calculated for $\mathrm{C}_{21} \mathrm{H}_{19} \mathrm{~F}_{3} \mathrm{~N}^{+}\left([\mathrm{M}+\mathrm{H}]^{+}\right)$: 342.1464, found: 332.1648 .

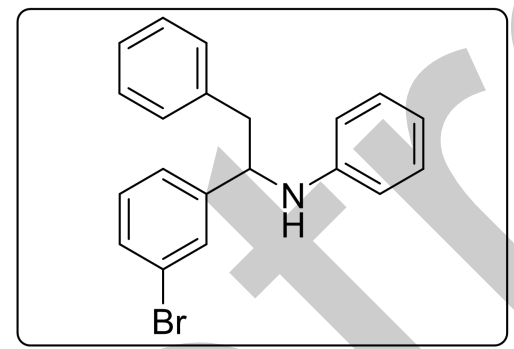

$N$-(1-(3-bromophenyl)-2-phenylethyl)aniline 3s

The reaction was carried out according to the general procedure on $0.2 \mathrm{mmol}$ scale (24 h). The residue was purified by flash column chromatography (petroleum ether: ethyl acetate, 100/1-50/1) to afford $3 \mathrm{~s},(65 \%, 45.6 \mathrm{mg})$, as a white solid. ${ }^{1} \mathrm{H}$ NMR (400 MHz, Chloroform- $d$ ) $\delta 7.41(\mathrm{t}, J=1.7 \mathrm{~Hz}, 1 \mathrm{H}), 7.28(\mathrm{~s}, 1 \mathrm{H}), 7.24-7.12(\mathrm{~m}, 4 \mathrm{H})$, $7.11-6.94(\mathrm{~m}, 5 \mathrm{H}), 6.57(\mathrm{t}, J=7.3 \mathrm{~Hz}, 1 \mathrm{H}), 6.35(\mathrm{~d}, J=8.6 \mathrm{~Hz}, 2 \mathrm{H}), 4.45(\mathrm{dd}, J=$ 8.3, 5.7 Hz, 1H), $3.03(\mathrm{dd}, J=14.0,5.7 \mathrm{~Hz}, 1 \mathrm{H}), 2.90(\mathrm{dd}, J=14.0,8.3 \mathrm{~Hz}, 1 \mathrm{H}) .{ }^{13} \mathrm{C}$ NMR (100 MHz, Chloroform-d) $\delta$ 145.9, 145.1, 136.1, 129.2, 129.1, 128.4, 128.1, 128.0, 127.6, 125.9, 124.1, 121.7, 116.8, 112.60, 57.9, 44.1. HRMS (ESI) Calculated for $\mathrm{C}_{20} \mathrm{H}_{19} \mathrm{BrN}^{+}\left([\mathrm{M}+\mathrm{H}]^{+}\right)$: 352.0695, found: 352.0697 . 


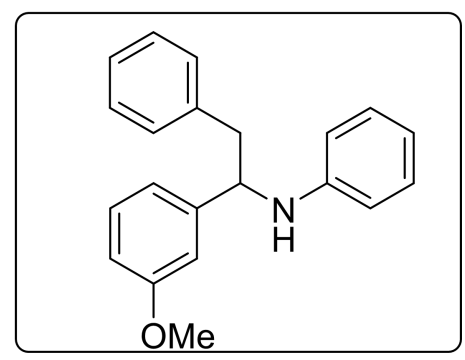

$\mathrm{N}$-(1-(3-methoxyphenyl)-2-phenylethyl)aniline 3t

The reaction was carried out according to the general procedure on $0.2 \mathrm{mmol}$ scale $(24 \mathrm{~h})$. The residue was purified by flash column chromatography (petroleum ether: ethyl acetate, 100/1-50/1) to afford 3s, (56\%, $33.9 \mathrm{mg})$, a colorless sticky oil. ${ }^{1} \mathrm{H}$ NMR (400 MHz, Chloroform-d) $\delta 7.34-7.00(\mathrm{~m}, 8 \mathrm{H}), 6.96-6.82(\mathrm{~m}, 2 \mathrm{H}), 6.77(\mathrm{dd}$, $J=7.8,2.9 \mathrm{~Hz}, 1 \mathrm{H}), 6.62(\mathrm{t}, J=7.8 \mathrm{~Hz}, 1 \mathrm{H}), 6.45(\mathrm{~d}, J=7.6 \mathrm{~Hz}, 2 \mathrm{H}), 4.54(\mathrm{dd}, J=$ 8.2, $5.7 \mathrm{~Hz}, 1 \mathrm{H}), 3.74$ (s, 3H), $3.12(\mathrm{dd}, J=14.0,5.7 \mathrm{~Hz}, 1 \mathrm{H}), 3.00$ (dd, $J=14.0,8.3$ $\mathrm{Hz}, 1 \mathrm{H}) .{ }^{13} \mathrm{C}$ NMR (100 MHz, Chloroform- $d$ ) $\delta$ 159.87, 147.32, 145.36, 137.70, $129.60,129.22,129.02,128.57,126.75,118.81,117.53,113.66,112.39,112.13,59.31$, 55.19, 45.12. HRMS (ESI) Calculated for $\mathrm{C}_{21} \mathrm{H}_{22} \mathrm{NO}^{+}\left([\mathrm{M}+\mathrm{H}]^{+}\right)$: 304.1696, found: 304.1696.

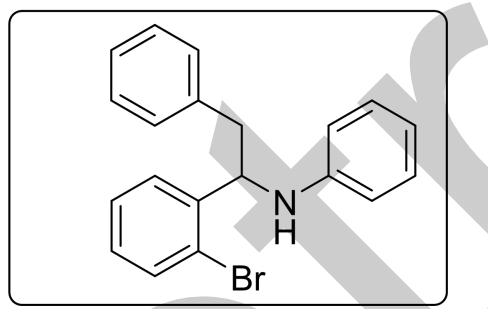

$N$-(1-(2-bromophenyl)-2-phenylethyl)aniline $3 \mathbf{u}$

The reaction was carried out according to the general procedure on $0.2 \mathrm{mmol}$ scale $(24 \mathrm{~h})$. The residue was purified by flash column chromatography (petroleum ether: ethyl acetate, 100/1-50/1) to afford 3u, (57\%, $40.0 \mathrm{mg})$, as a white solid. ${ }^{1} \mathrm{H}$ NMR $(400 \mathrm{MHz}$, Chloroform- $d$ ) $\delta 7.59(\mathrm{~d}, J=7.0 \mathrm{~Hz}, 1 \mathrm{H}), 7.43(\mathrm{~d}, J=7.7 \mathrm{~Hz}, 1 \mathrm{H}), 7.32-$ $7.21(\mathrm{~m}, 6 \mathrm{H}), 7.12(\mathrm{~d}, J=6.1 \mathrm{~Hz}, 1 \mathrm{H}), 7.03(\mathrm{t}, J=7.9 \mathrm{~Hz}, 2 \mathrm{H}), 6.61$ (t, $J=7.3 \mathrm{~Hz}$, 1H), $6.36(\mathrm{~d}, J=7.7 \mathrm{~Hz}, 2 \mathrm{H}), 4.99$ (dd, $J=9.5,3.9 \mathrm{~Hz}, 1 \mathrm{H}), 3.30$ (dd, $J=14.2,3.9$ $\mathrm{Hz}, 1 \mathrm{H}), 2.75(\mathrm{dd}, J=14.2,9.5 \mathrm{~Hz}, 1 \mathrm{H}) .{ }^{13} \mathrm{C}$ NMR $(100 \mathrm{MHz}$, Chloroform- $d$ ) $\delta 146.8$, 141.8, 137.6, 133.1, 129.1 (2C), 128.7 (2C), 127.9 (2C), 126.9, 122.9, 117.7, 113.6, 58.1, 42.8. HRMS (ESI) Calculated for $\mathrm{C}_{20} \mathrm{H}_{19} \mathrm{BrN}^{+}\left([\mathrm{M}+\mathrm{H}]^{+}\right)$: 352.0695, found: 352.0697 . 


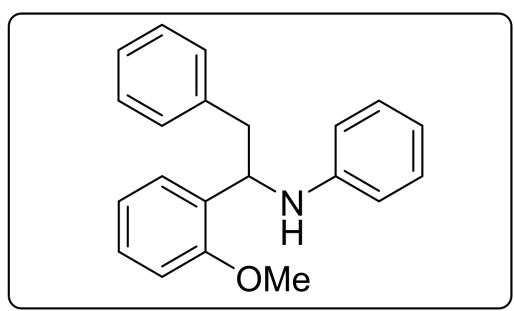

$\mathrm{N}$-(1-(2-methoxyphenyl)-2-phenylethyl)aniline 3v

The reaction was carried out according to the general procedure on $0.2 \mathrm{mmol}$ scale (36 h). The residue was purified by flash column chromatography (petroleum ether: ethyl acetate, 100/1-50/1) to afford $\mathbf{3 v}$, (48\%, $29.2 \mathrm{mg})$, a colorless sticky oil. ${ }^{1} \mathrm{H}$ NMR (400 MHz, Chloroform-d) $\delta 7.31-7.11(\mathrm{~m}, 7 \mathrm{H}), 7.07-6.99(\mathrm{~m}, 2 \mathrm{H}), 6.87(\mathrm{dd}$, $J=15.6,7.6 \mathrm{~Hz}, 2 \mathrm{H}), 6.59(\mathrm{t}, J=7.8 \mathrm{~Hz}, 1 \mathrm{H}), 6.45(\mathrm{~d}, J=9.5 \mathrm{~Hz}, 2 \mathrm{H}), 4.99$ (dd, $J=$ 8.1, 5.1 Hz, 1H), 3.87 (s, 3H), $3.21(\mathrm{dd}, J=13.9,5.1 \mathrm{~Hz}, 1 \mathrm{H}), 2.89$ (dd, $J=13.9,8.2$ Hz, 1H). ${ }^{13} \mathrm{C}$ NMR (100 MHz, Chloroform- $d$ ) $\delta$ 156.77, 147.39, 138.63, 130.92, $129.23,129.00,128.35,127.89$, 127.19, 126.42, 120.72, 117.19, 113.51, 110.45, 55.39, 53.67, 42.65. HRMS (ESI) Calculated for $\mathrm{C}_{21} \mathrm{H}_{22} \mathrm{NO}^{+}\left([\mathrm{M}+\mathrm{H}]^{+}\right)$: 304.1696, found: 304.1699 .

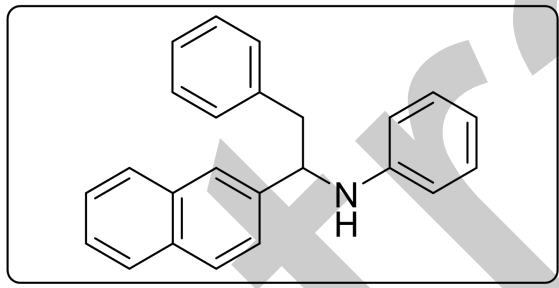

$N$-(1-(naphthalen-2-yl)-2-phenylethyl)aniline 3w

The reaction was carried out according to the general procedure on $0.2 \mathrm{mmol}$ scale $(24 \mathrm{~h})$. The residue was purified by flash column chromatography (petroleum ether: ethyl acetate, 100/1-50/1) to afford 3w, $(81 \%, 52.3 \mathrm{mg})$, as a green solid. ${ }^{1} \mathrm{H}$ NMR $(400 \mathrm{MHz}$, Chloroform- $d$ ) $\delta 7.87-7.72(\mathrm{~m}, 4 \mathrm{H}), 7.52-7.39(\mathrm{~m}, 3 \mathrm{H}), 7.30-7.18(\mathrm{~m}$, $3 \mathrm{H}), 7.14(\mathrm{~d}, J=8.0 \mathrm{~Hz}, 2 \mathrm{H}), 7.02(\mathrm{t}, J=7.9 \mathrm{~Hz}, 2 \mathrm{H}), 6.60(\mathrm{t}, J=7.3 \mathrm{~Hz}, 1 \mathrm{H}), 6.48$ $(\mathrm{d}, J=7.7 \mathrm{~Hz}, 2 \mathrm{H}), 4.74(\mathrm{dd}, J=8.2,5.7 \mathrm{~Hz}, 1 \mathrm{H}), 3.22(\mathrm{dd}, J=14.0,5.6 \mathrm{~Hz}, 1 \mathrm{H})$, 3.07 (dd, $J=14.0,8.3 \mathrm{~Hz}, 1 \mathrm{H}) .{ }^{13} \mathrm{C}$ NMR (100 MHz, Chloroform- $d$ ) $\delta$ 147.3, 141.1, $137.6,133.6$, 132.9, 129.3, 129.1, 128.6, 128.5, 127.9, 127.7, 126.8, 126.0, 125.6, 125.2, 124.8, 117.6, 113.8, 59.5, 45.2. HRMS (ESI) Calculated for $\mathrm{C}_{24} \mathrm{H}_{22} \mathrm{~N}^{+}$ $\left([\mathrm{M}+\mathrm{H}]^{+}\right): 324.1747$, found: 324.1749 . 


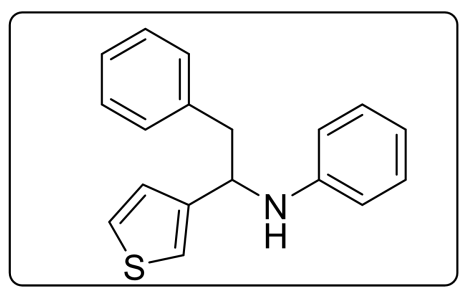

$N$-(2-phenyl-1-(thiophen-3-yl)ethyl)aniline 3x

The reaction was carried out according to the general procedure on $0.2 \mathrm{mmol}$ scale (24 h). The residue was purified by flash column chromatography (petroleum ether: ethyl acetate, 100/1-40/1) to afford 3x, (48\%, $26.8 \mathrm{mg})$, a colorless sticky oil. ${ }^{1} \mathrm{H}$ NMR (400 MHz, Chloroform-d) $\delta 7.33-7.16(\mathrm{~m}, 4 \mathrm{H}), 7.13-7.06(\mathrm{~m}, 4 \mathrm{H}), 7.03-$ $6.99(\mathrm{~m}, 2 \mathrm{H}), 6.65(\mathrm{t}, J=7.3 \mathrm{~Hz}, 1 \mathrm{H}), 6.52(\mathrm{~d}, J=8.6 \mathrm{~Hz}, 2 \mathrm{H}), 4.73(\mathrm{t}, J=6.7 \mathrm{~Hz}$, 1H), 3.17 - 3.05 (m, 2H). ${ }^{13} \mathrm{C}$ NMR (100 MHz, Chloroform-d) $\delta$ 147.25, 144.64, $137.59,129.31,129.12,128.48,126.70,126.16,125.95,121.01,117.64,113.61$, 55.24, 43.88. HRMS (ESI) Calculated for $\mathrm{C}_{18} \mathrm{H}_{18} \mathrm{NS}^{+}\left([\mathrm{M}+\mathrm{H}]^{+}\right)$: 280.1154, found: 280.1156.
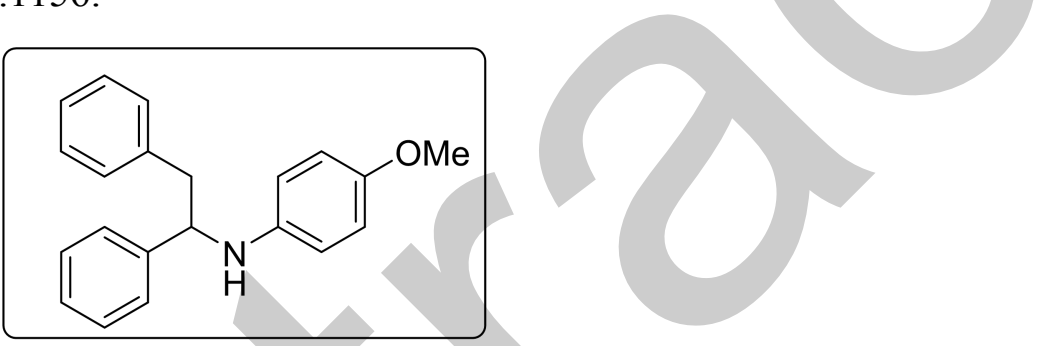

$N$-(1,2-diphenylethyl)-4-methoxyaniline $3 \mathbf{y}$

The reaction was carried out according to the general procedure on $0.2 \mathrm{mmol}$ scale $(36 \mathrm{~h})$. The residue was purified by flash column chromatography (petroleum ether: ethyl acetate, 100/1-50/1) to afford $\mathbf{3 y}$, (53\%, $32.1 \mathrm{mg})$, a colorless sticky oil. ${ }^{1} \mathrm{H}$ NMR (400 MHz, Chloroform- $d$ ) $\delta 7.37-7.18(\mathrm{~m}, 8 \mathrm{H}), 7.12(\mathrm{~d}, J=6.7 \mathrm{~Hz}, 2 \mathrm{H}), 6.64$ $(\mathrm{d}, J=9.0 \mathrm{~Hz}, 2 \mathrm{H}), 6.40(\mathrm{~d}, J=8.9 \mathrm{~Hz}, 2 \mathrm{H}), 4.50(\mathrm{dd}, J=8.3,5.7 \mathrm{~Hz}, 1 \mathrm{H}), 3.66(\mathrm{~s}$, $3 \mathrm{H}), 3.11(\mathrm{dd}, J=13.9,5.7 \mathrm{~Hz}, 1 \mathrm{H}), 2.99(\mathrm{dd}, J=13.9,8.3 \mathrm{~Hz}, 1 \mathrm{H}) .{ }^{13} \mathrm{C}$ NMR $(100$ MHz, Chloroform-d) $\delta 152.1,143.7,141.5,137.8,129.2$, 128.6, 128.5, 127.0, 126.7, 126.5, 114.9, 114.7, 60.1, 55.7, 45.3. HRMS (ESI) Calculated for $\mathrm{C}_{21} \mathrm{H}_{22} \mathrm{NO}^{+}$ $\left([\mathrm{M}+\mathrm{H}]^{+}\right): 304.1696$, found: 304.1698 . 


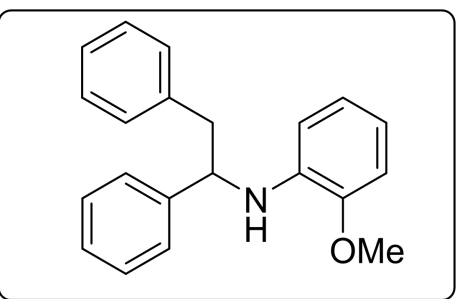

$N$-(1,2-diphenylethyl)-2-methoxyaniline $3 z$

The reaction was carried out according to the general procedure on $0.2 \mathrm{mmol}$ scale (36 h). The residue was purified by flash column chromatography (petroleum ether: ethyl acetate, 100/1-50/1) to afford $\mathbf{3 z},(66 \%, 40.0 \mathrm{mg})$, a colorless sticky oil. ${ }^{1} \mathrm{H}$ NMR (400 MHz, Chloroform-d) $\delta 7.44-6.98(\mathrm{~m}, 10 \mathrm{H}), 6.80-6.41(\mathrm{~m}, 3 \mathrm{H}), 6.32(\mathrm{~d}$, $J=8.7 \mathrm{~Hz}, 1 \mathrm{H}), 4.53(\mathrm{t}, J=6.9 \mathrm{~Hz}, 1 \mathrm{H}), 3.80(\mathrm{~s}, 3 \mathrm{H}), 3.16-3.06(\mathrm{~m}, 2 \mathrm{H}) .{ }^{13} \mathrm{C} \mathrm{NMR}$ (100 MHz, Chloroform-d) $\delta 146.89,143.58,137.99,137.34,129.34,128.50,128.38$, 126.99, 126.54, 121.15, 116.51, 111.26, 109.52, 59.51, 55.62, 45.31. HRMS (ESI) Calculated for $\mathrm{C}_{21} \mathrm{H}_{22} \mathrm{NO}^{+}\left([\mathrm{M}+\mathrm{H}]^{+}\right)$: 304.1696, found: 304.1699 .

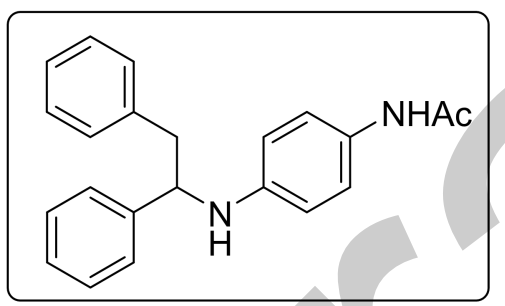

$N$-(4-((1,2-diphenylethyl)amino)phenyl)acetamide 3aa

The reaction was carried out according to the general procedure on $0.2 \mathrm{mmol}$ scale (36 h). The residue was purified by flash column chromatography (petroleum ether: ethyl acetate, 100/1-40/1) to afford 3aa, (57\%, $37.6 \mathrm{mg})$, as a white solid. ${ }^{1} \mathrm{H}$ NMR $(400 \mathrm{MHz}$, Chloroform- $d$ ) $\delta 7.40-7.18(\mathrm{~m}, 8 \mathrm{H}), 7.10(\mathrm{~d}, J=8.8 \mathrm{~Hz}, 4 \mathrm{H}), 6.38(\mathrm{~d}, J=$ $8.8 \mathrm{~Hz}, 2 \mathrm{H}), 4.54(\mathrm{dd}, J=8.1,5.8 \mathrm{~Hz}, 1 \mathrm{H}), 3.11(\mathrm{dd}, J=14.0,5.6 \mathrm{~Hz}, 1 \mathrm{H}), 2.99(\mathrm{dd}, J$ $=14.0,8.2 \mathrm{~Hz}, 1 \mathrm{H}), 2.03(\mathrm{~s}, 3 \mathrm{H}) .{ }^{13} \mathrm{C}$ NMR (100 MHz, Chloroform- $d$ ) $\delta$ 168.19, $144.49,143.30,137.63,129.20,128.59,128.56,128.20,127.12,126.75,126.47$, 122.23, 113.84, 59.41, 45.10, 24.18. HRMS (ESI) Calculated for $\mathrm{C}_{22} \mathrm{H}_{23} \mathrm{~N}_{2} \mathrm{O}^{+}$ $\left([\mathrm{M}+\mathrm{H}]^{+}\right): 331.1805$, found: 331.1808 . 


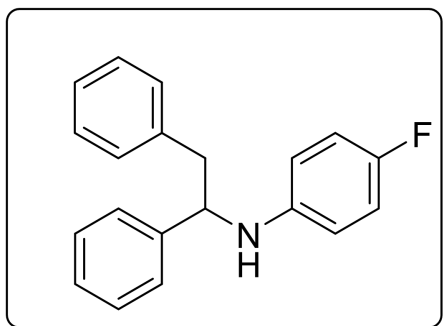

$N$-(1,2-diphenylethyl)-4-fluoroaniline 3bb

The reaction was carried out according to the general procedure on $0.2 \mathrm{mmol}$ scale (24 h). The residue was purified by flash column chromatography (petroleum ether: ethyl acetate, 100/1-50/1) to afford $\mathbf{3 b b},(77 \%, 44.8 \mathrm{mg})$, a colorless sticky oil. ${ }^{1} \mathrm{H}$ NMR (400 MHz, Chloroform- $d$ ) $\delta 7.34-7.18(\mathrm{~m}, 8 \mathrm{H}), 7.11(\mathrm{~d}, J=6.8 \mathrm{~Hz}, 2 \mathrm{H}), 6.73$ (t, $J=8.8 \mathrm{~Hz}, 2 \mathrm{H}), 6.36(\mathrm{dd}, J=8.2,5.2 \mathrm{~Hz}, 2 \mathrm{H}), 4.50(\mathrm{dd}, J=8.1,5.8 \mathrm{~Hz}, 1 \mathrm{H}), 3.12$ $(\mathrm{dd}, J=14.0,5.6 \mathrm{~Hz}, 1 \mathrm{H}), 2.98(\mathrm{dd}, J=14.0,8.3 \mathrm{~Hz}, 1 \mathrm{H}) \cdot{ }^{13} \mathrm{C}$ NMR $(100 \mathrm{MHz}$, Chloroform-d) $\delta 155.82(\mathrm{~d}, J=235.1 \mathrm{~Hz}), 143.62,143.26,137.62,129.21,128.64$, 128.60, 127.19, 126.80, 126.46, 115.44 (d, $J=22.3 \mathrm{~Hz}), 114.47$ (d, $J=7.4 \mathrm{~Hz}), 59.84$, 45.24. HRMS (ESI) Calculated for $\mathrm{C}_{20} \mathrm{H}_{19} \mathrm{FN}^{+}\left([\mathrm{M}+\mathrm{H}]^{+}\right)$: 292.1496, found: 292.1497 .

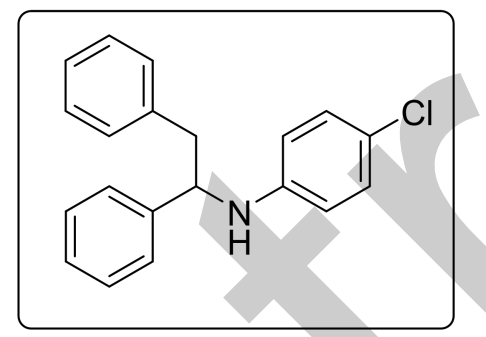

4-chloro- $N$-(1,2-diphenylethyl)aniline 3cc

The reaction was carried out according to the general procedure on $0.2 \mathrm{mmol}$ scale $(24 \mathrm{~h})$. The residue was purified by flash column chromatography (petroleum ether: ethyl acetate, 100/1-50/1) to afford 3cc, $(80 \%, 49.1 \mathrm{mg})$, a colorless sticky oil. ${ }^{1} \mathrm{H}$ NMR (400 MHz, Chloroform- $d$ ) $\delta 7.34-7.11(\mathrm{~m}, 8 \mathrm{H}), 7.03(\mathrm{~d}, J=8.1 \mathrm{~Hz}, 2 \mathrm{H}), 6.90$ $(\mathrm{d}, J=8.9 \mathrm{~Hz}, 2 \mathrm{H}), 6.28(\mathrm{~d}, J=8.9 \mathrm{~Hz}, 2 \mathrm{H}), 4.46(\mathrm{dd}, J=8.2,5.7 \mathrm{~Hz}, 1 \mathrm{H}), 3.06(\mathrm{dd}$, $J=14.0,5.7 \mathrm{~Hz}, 1 \mathrm{H}), 2.92(\mathrm{dd}, J=14.0,8.2 \mathrm{~Hz}, 1 \mathrm{H}) .{ }^{13} \mathrm{C} \mathrm{NMR}(100 \mathrm{MHz}$, Chloroform- $d$ ) $\delta 144.7,141.8,136.4,128.1,127.8,127.6,127.6,126.2,125.8,125.3$, 121.1, 113.7, 58.2, 44.0 HRMS (ESI) Calculated for $\mathrm{C}_{20} \mathrm{H}_{19} \mathrm{ClN}^{+}\left([\mathrm{M}+\mathrm{H}]^{+}\right): 308.1201$, found: 308.1203 . 


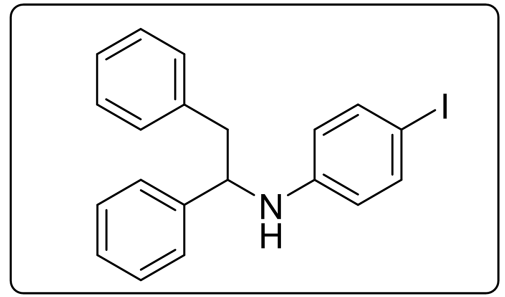

$N$-(1,2-diphenylethyl)-4-iodoaniline 3dd

The reaction was carried out according to the general procedure on $0.2 \mathrm{mmol}$ scale (24 h). The residue was purified by flash column chromatography (petroleum ether: ethyl acetate, 100/1-50/1) to afford 3dd, (53\%, $42.4 \mathrm{mg})$, as a white solid. ${ }^{1} \mathrm{H}$ NMR $(400 \mathrm{MHz}$, Chloroform- $d) \delta 7.29-7.11(\mathrm{~m}, 10 \mathrm{H}), 7.06-6.97(\mathrm{~m}, 2 \mathrm{H}), 6.15(\mathrm{~d}, J=$ $8.9 \mathrm{~Hz}, 2 \mathrm{H}), 4.46(\mathrm{dd}, J=8.3,5.7 \mathrm{~Hz}, 1 \mathrm{H}), 3.06(\mathrm{dd}, J=14.0,5.7 \mathrm{~Hz}, 1 \mathrm{H}), 2.91(\mathrm{dd}$, $J=14.0,8.3 \mathrm{~Hz}, 1 \mathrm{H}) .{ }^{13} \mathrm{C}$ NMR (100 MHz, Chloroform- $d$ ) $\delta$ 146.8, 142.8, 137.6, 137.4, 129.2, 128.7, 128.6, 127.3, 126.9, 126.4, 115.9, 78.3, 59.0, 45.0. HRMS (ESI) Calculated for $\mathrm{C}_{20} \mathrm{H}_{19} \mathrm{IN}^{+}\left([\mathrm{M}+\mathrm{H}]^{+}\right)$: 400.0557, found: 400.0559 .

\section{A large-scale synthesis by utilizing continuous-flow micro-tubing reactors}

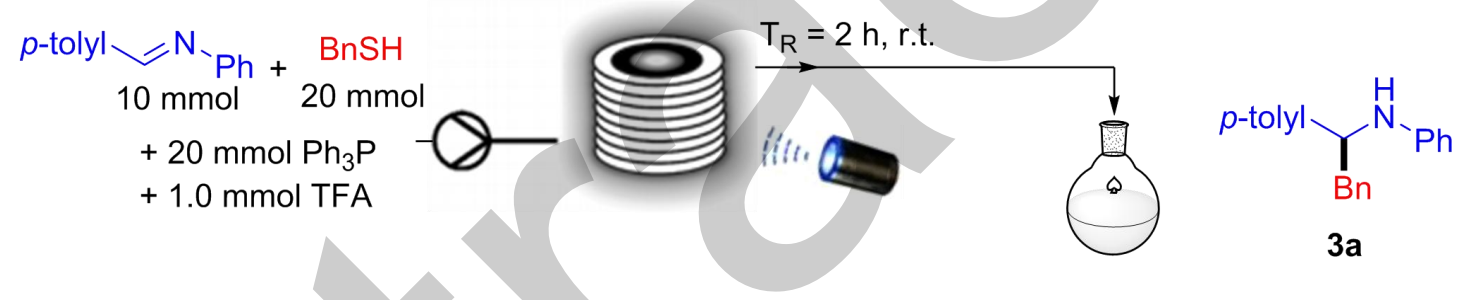

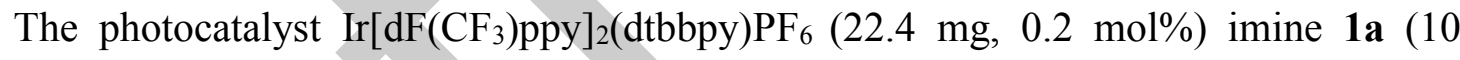
mmol, 1.95 g, 1.0 equiv.), $\mathrm{Ph}_{3} \mathrm{P}$ (5.24 g, 20 mmol, 2.0 equiv.), Benzyl thiol (20 mmol, $2.48 \mathrm{~g}, 2.0$ equiv.) and TFA (115 $\mathrm{mg}, 10 \mathrm{~mol} \%$ ) were added to a $250 \mathrm{~mL}$ flask be quipped with a rubber septum containing $100 \mathrm{~mL}$ DMA and sparged with argon for 20 minutes. An Syrris Asia pump was filled with the combined organic reaction mixture connected to the flow reactor. The reaction mixture would then flow into the micro-tubing reactor was irradiated with $90 \mathrm{~W}$ blue LED. Flow rate of the combined organic mixture were set at the speed of $50 \mathrm{~mL} / \mathrm{h}$. When the reaction finished, the mixture was quenched with water and extracted with ethyl acetate $(3 \times 60 \mathrm{~mL})$. The organic layers were combined and concentrated under vacuo. The product was purified by flash column chromatography on silica gel (petroleum ether: ethyl acetate)afford 3a, (72\%, $2.07 \mathrm{~g})$ a colorless sticky oil. 


\section{Mechanistic Investigations}

\section{Control Experiment with Additives}

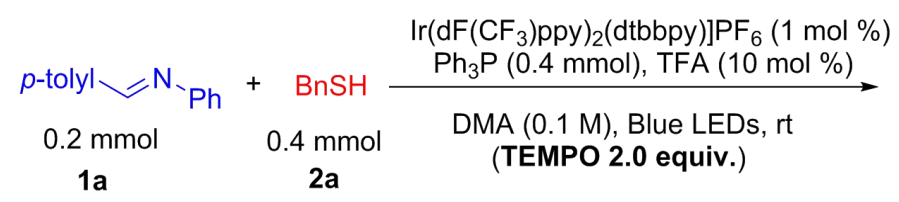
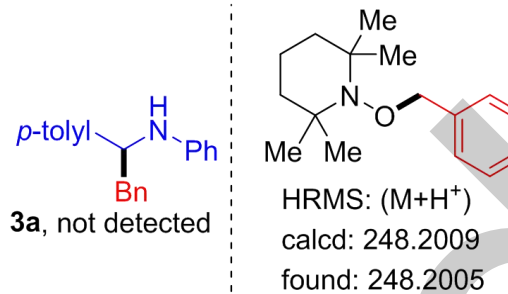

To a $10 \mathrm{~mL}$ Schlenk tube equipped with a magnetic stir bar was added imine $\mathbf{1 a}(0.2$ mmol, $39.0 \mathrm{mg}$, 1.0 equiv.), photocatalyst $\operatorname{Ir}\left[\mathrm{dF}\left(\mathrm{CF}_{3}\right) \text { ppy }\right]_{2}(\mathrm{dtbbpy}) \mathrm{PF}_{6}(2.3 \mathrm{mg}, 1$ mol\%), $\mathrm{Ph}_{3} \mathrm{P}$ (104.8 mg, $0.4 \mathrm{mmol}, 2.0$ equiv.) and additive (TEMPO $0.4 \mathrm{mmol}, 2$ equiv.), and the tube was evacuated and backfilled with Ar (three times). Benzyl thiol (0.4 mmol, $49.6 \mathrm{mg}, 2.0$ equiv.) and TFA (2.3 mg, $10 \mathrm{~mol} \%)$ in DMA (2.0 mL) were added by syringe under Ar. The tube was then sealed and was placed at a distance (app. $5 \mathrm{~cm}$ ) from $5 \mathrm{~W}$ blue LEDs lamp, and the mixture was stirred for $36 \mathrm{~h}$ at room temperature. The corresponding amine product 3a was not detected according to both TLC and GC-Mass analysis. The product 1-(benzyloxy)-2,2,6,6-tetramethylpiperidine was detected by ESI-HRMS.

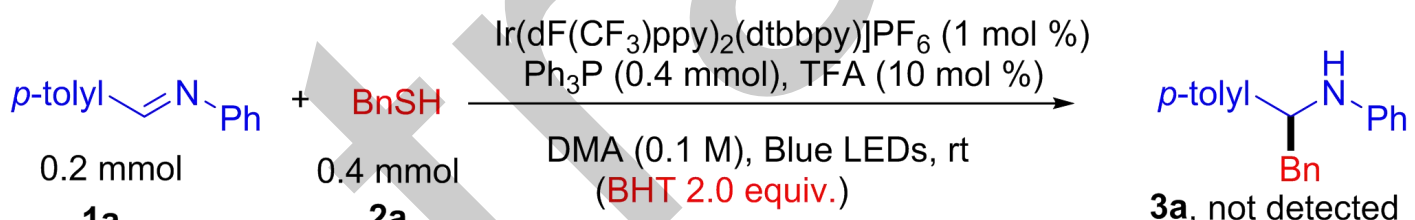

To a $10 \mathrm{~mL}$ Schlenk tube equipped with a magnetic stir bar was added imine 1a $(0.2$ mmol, $39.0 \mathrm{mg}, 1.0$ equiv.), photocatalyst $\operatorname{Ir}\left[\mathrm{dF}\left(\mathrm{CF}_{3}\right) \text { ppy }\right]_{2}(\mathrm{dtbbpy}) \mathrm{PF}_{6}(2.3 \mathrm{mg}, 1$ mol\%), $\mathrm{Ph}_{3} \mathrm{P}$ (104.8 mg, $0.4 \mathrm{mmol}, 2.0$ equiv.) and additive (BHT $0.4 \mathrm{mmol}, 2$ equiv.), and the tube was evacuated and backfilled with Ar (three times). Benzyl thiol (0.4 mmol, $49.6 \mathrm{mg}, 2.0$ equiv. $)$ and TFA (2.3 mg, $10 \mathrm{~mol} \%)$ in DMA (2.0 mL) were added by syringe under Ar. The tube was then sealed and was placed at a distance (app. $5 \mathrm{~cm}$ ) from $5 \mathrm{~W}$ blue LEDs lamp, and the mixture was stirred for $36 \mathrm{~h}$ at room temperature. The corresponding amine product 3a was not detected according to both TLC and GC-Mass analysis, which was fully inhibited by BHT. Therefore, an electron-transfer-triggered radical pathway should be involved in this transformation. 


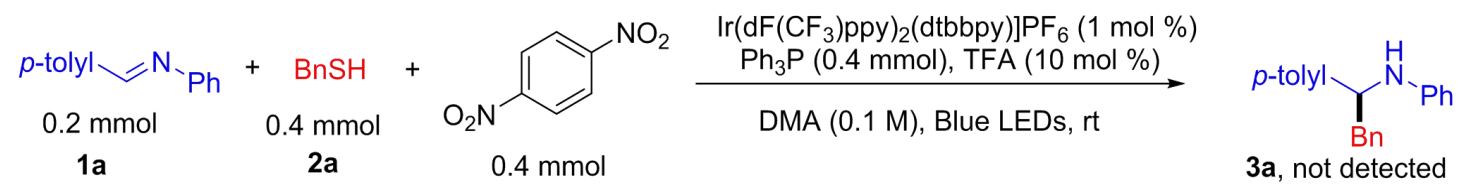

To a $10 \mathrm{~mL}$ Schlenk tube equipped with a magnetic stir bar was added imine 1a $(0.2$ mmol, $39.0 \mathrm{mg}$, 1.0 equiv.), photocatalyst $\operatorname{Ir}\left[\mathrm{dF}\left(\mathrm{CF}_{3}\right) \text { ppy }\right]_{2}(\mathrm{dtbbpy}) \mathrm{PF}_{6}(2.3 \mathrm{mg}, 1$ mol\%), $\mathrm{Ph}_{3} \mathrm{P}$ (104.8 mg, $0.4 \mathrm{mmol}, 2.0$ equiv.) and additive (1,4-dinitrobenzene, 0.4 mmol, 2 equiv.), and the tube was evacuated and backfilled with Ar (three times). Benzyl thiol (0.4 mmol, $49.6 \mathrm{mg}, 2.0$ equiv.) and TFA (2.3 mg, $10 \mathrm{~mol} \%)$ in DMA $(2.0 \mathrm{~mL})$ were added by syringe under Ar. The tube was then sealed and was placed at a distance (app. $5 \mathrm{~cm}$ ) from $5 \mathrm{~W}$ blue LEDs lamp, and the mixture was stirred for $36 \mathrm{~h}$ at room temperature. The product 3a could not be formed. The reaction was fully inhibited by 1,4-dinitrobenzne. Therefore, an electron-transfer-triggered radical pathway should be involved in this transformation.

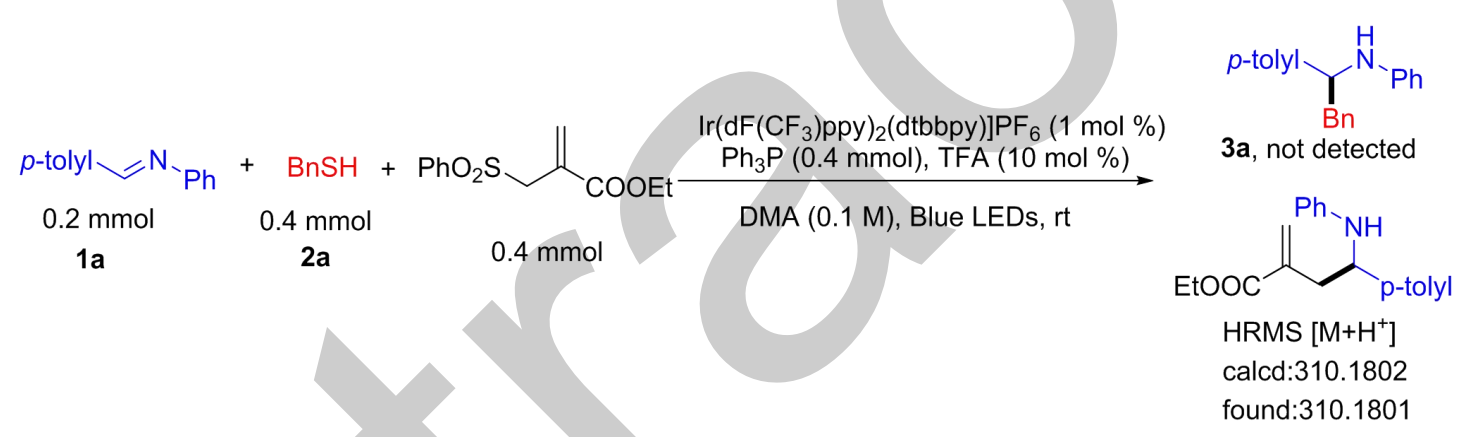

To a $10 \mathrm{~mL}$ Schlenk tube equipped with a magnetic stir bar was added imine 1a $(0.2$ mmol, $39.0 \mathrm{mg}, 1.0$ equiv.), photocatalyst $\operatorname{Ir}\left[\mathrm{dF}\left(\mathrm{CF}_{3}\right) \text { ppy }\right]_{2}(\mathrm{dtbbpy}) \mathrm{PF}_{6}(2.3 \mathrm{mg}, 1$ mol\%), $\mathrm{Ph}_{3} \mathrm{P} \quad(104.8 \mathrm{mg}, \quad 0.4 \mathrm{mmol}, 2.0$ equiv.) and additive (2-((phenylsulfonyl)methyl)acrylate, $0.4 \mathrm{mmol}, 2$ equiv.), and the tube was evacuated and backfilled with Ar (three times). Benzyl thiol (0.4 mmol, $49.6 \mathrm{mg}, 2.0$ equiv.) and TFA (2.3 mg, $10 \mathrm{~mol} \%)$ in DMA (2.0 mL) were added by syringe under Ar. The tube was then sealed and was placed at a distance (app. $5 \mathrm{~cm}$ ) from $5 \mathrm{~W}$ blue LEDs lamp, and the mixture was stirred for $36 \mathrm{~h}$ at room temperature. The product $\mathbf{3 a}$ could not be formed. $34 \mathrm{~h}$ later, the reaction mixture was sent for HRMS analysis, the compound ethyl 2-methylene-4-(phenylamino)-4-(p-tolyl)butanoate were detected by HRMS. It was envisaged that this compound was generated by addition of $\alpha$-amino alkyl radical. 


\section{Luminescence Quenching Experiment}

The luminescence quenching experiment was taken using a Cary Eclipse fluorescence spectrophotometer (Varian, USA). The experiments were carried out in $2.5 \times 10^{-6}$ $\mathrm{mol} / \mathrm{L}$ of $\left[\operatorname{Ir}\left\{\mathrm{dF}\left(\mathrm{CF}_{3}\right) \mathrm{ppy}\right\}_{2}\{\mathrm{dtbbpy}\}\right] \mathrm{PF}_{6}$ in DMA at $25{ }^{\circ} \mathrm{C}$. The emission intensity was collected at $475 \mathrm{~nm}$. The concentrations of quenchers (1a, 2a and $\mathrm{Ph}_{3} \mathrm{P}$ ) in solvent were $0,2,4,6,10,14,24 \mathrm{mM}$.
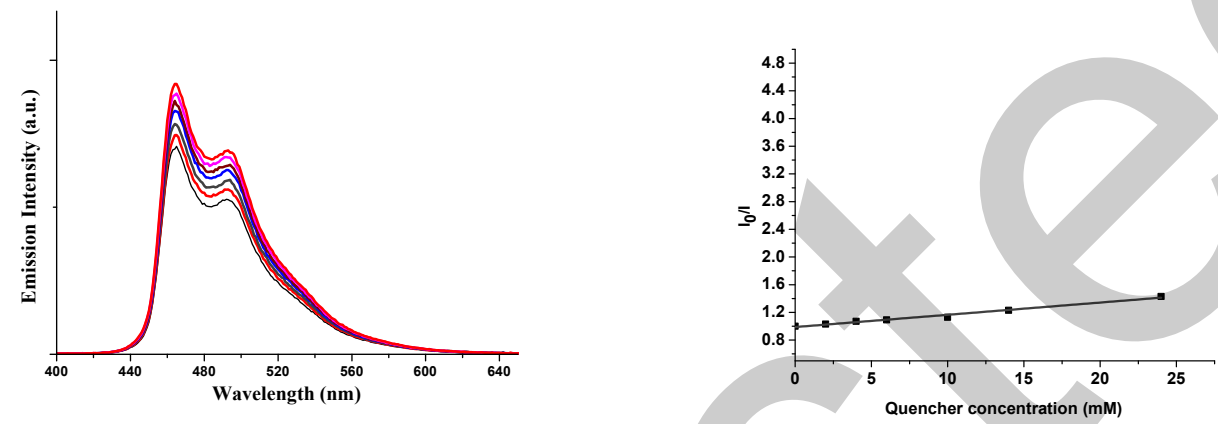

Luminescence quenching of $\left[\operatorname{Ir}\left\{\mathrm{dF}\left(\mathrm{CF}_{3}\right) \mathrm{ppy}_{2}\{\mathrm{dtbbpy}\}\right] \mathrm{PF}_{6}\right.$ by $\mathbf{1 a}$
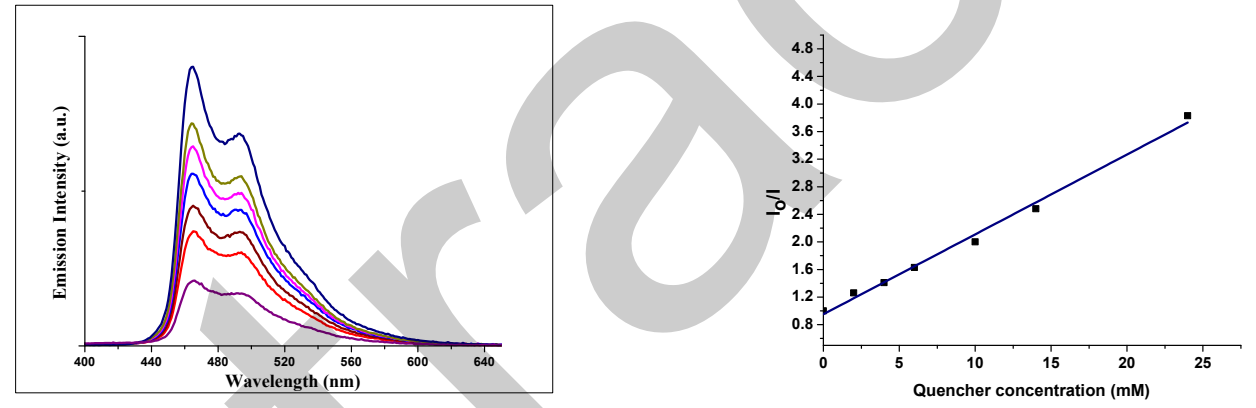

Luminescence quenching of $\left[\operatorname{Ir}\left\{\mathrm{dF}\left(\mathrm{CF}_{3}\right) \text { ppy }\right\}_{2}\{\mathrm{dtbbpy}\}\right] \mathrm{PF}_{6}$ by $\mathbf{2 a}$
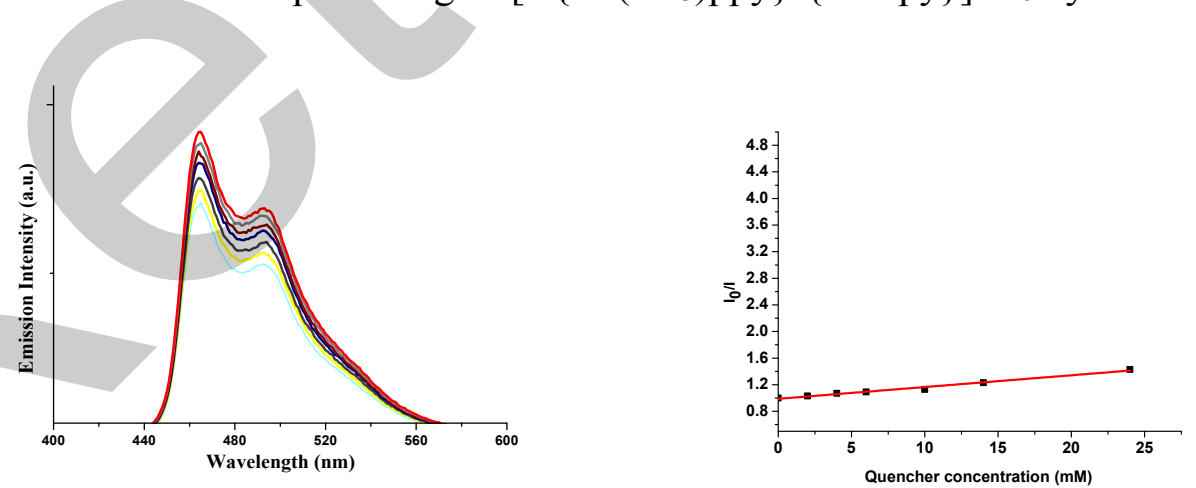

Luminescence quenching of $\left[\operatorname{Ir}\left\{\mathrm{dF}\left(\mathrm{CF}_{3}\right) \text { ppy }\right\}_{2}\{\mathrm{dtbbpy}\}\right] \mathrm{PF}_{6}$ by $\mathrm{Ph}_{3} \mathrm{P}$

\section{Quantum yield measurement}

The quantum yield $(\phi)$ was determined by the known ferrioxalate actinometry method.

A ferrioxalate actinometry solution was prepared by following the Hammond 
variation of the Hatchard and Parker procedure outlined in Handbook of Photochemistry. ${ }^{2}$ The irradiated light intensity was estimated to $8.00 \times 10^{-9}$ einstein $\mathrm{S}^{-1}$ by using $\mathrm{K}_{3}\left[\mathrm{Fe}\left(\mathrm{C}_{2} \mathrm{O}_{4}\right)_{3}\right]$ as an actinometer. For five clean tubes, according to the general procedure, the $0.1 \mathrm{mmol}$ scale model reaction solution was irradiated with 5 W blue LEDs for specified time intervals (10 min, $15 \mathrm{~min}, 20 \mathrm{~min}, 25 \mathrm{~min}$ and 30 min). The moles of products formed were determined by ${ }^{1} \mathrm{H}$ NMR measurement with 1,3,5-trimethoxylbenzene as reference standard. The number of moles of products ( $\mathrm{y}$ axis) per unit time is related to the number of photons ( $\mathrm{x}$ axis, calculated from the light intensity). The slope gives the quantum yield $(\Phi)$ of the photo reaction, 0.41 .

\section{Starting Materials}

General Procedure for preparation of imines: A solution of aryl amines ( $7.5 \mathrm{mmol})$ and substituted benzaldehydes $(5.0 \mathrm{mmol})$ in $\mathrm{CH}_{2} \mathrm{Cl}_{2}(20 \mathrm{~mL})$ was added $\mathrm{MgSO}_{4}(2.0$ $\mathrm{g})$, the reaction mixture was stirred at room temperature overnight. The reaction mixture was then filtrated and purified by column chromatography to give pure substituted imines. ${ }^{1}$

\section{Copies of ${ }^{1} \mathrm{H}$ NMR, ${ }^{13} \mathrm{C}$ NMR Spectra}

N-(2-phenyl-1-(p-tolyl)ethyl)aniline 3a

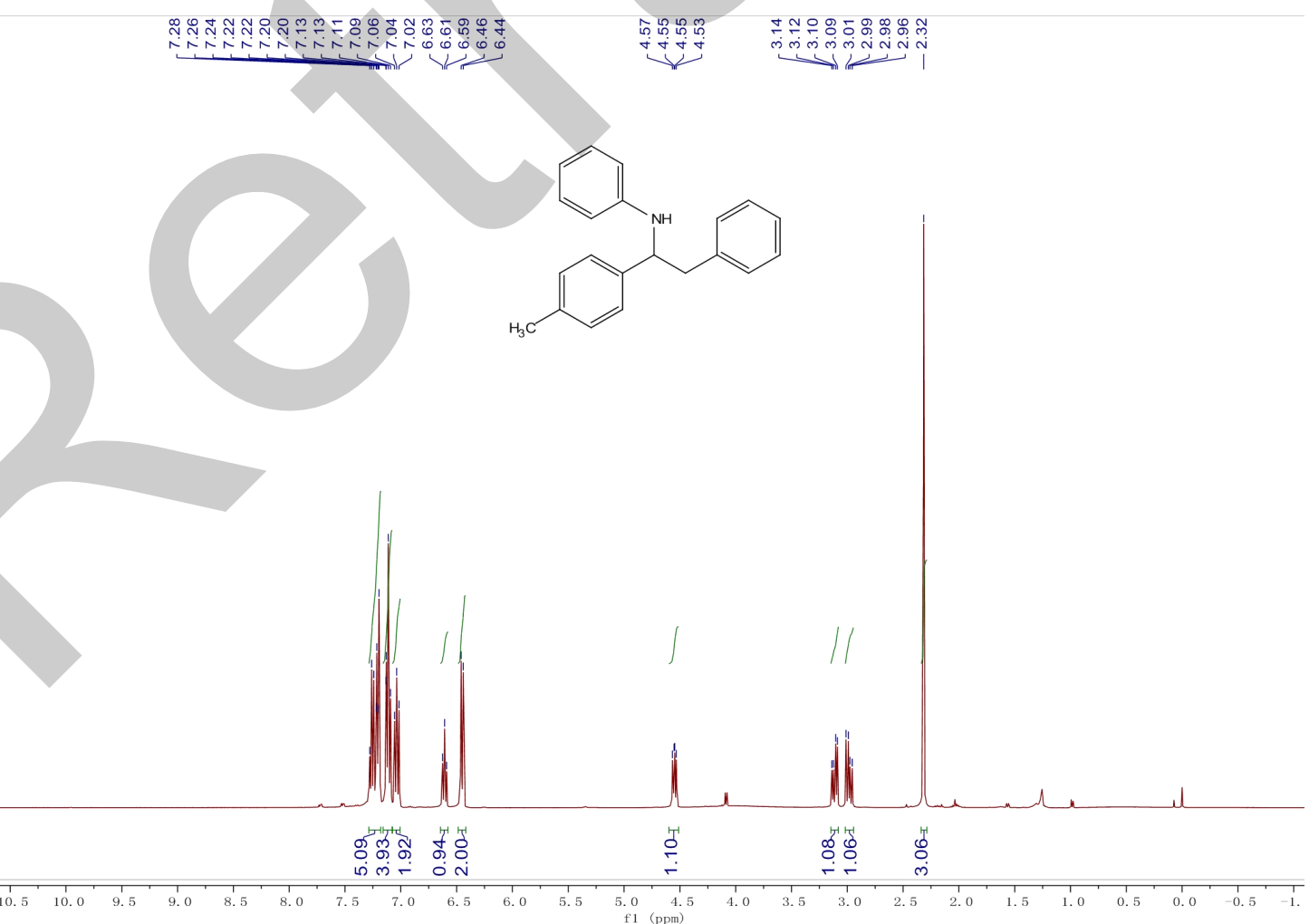




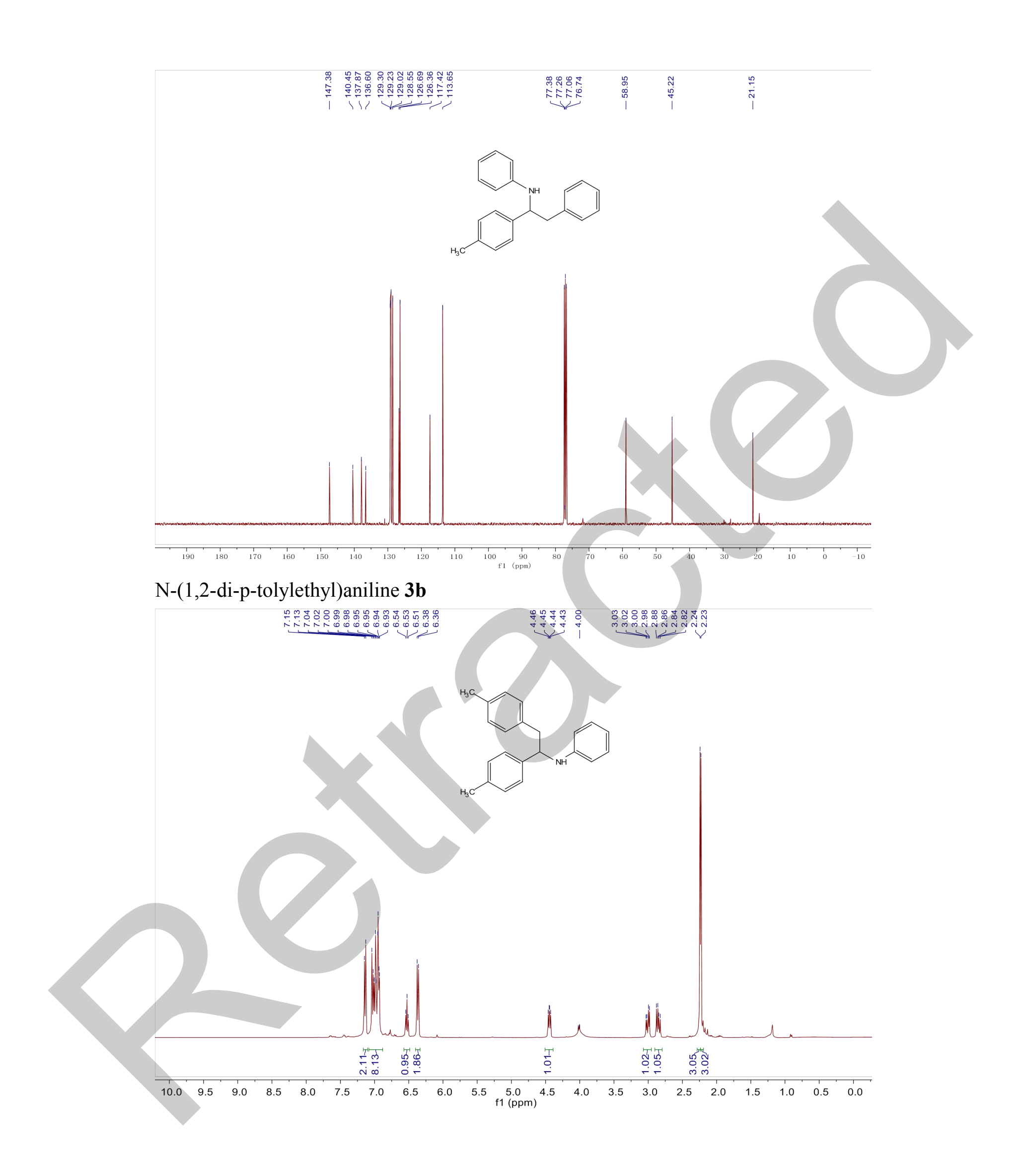




$$
\frac{e^{e^{2}}}{e^{e}}
$$




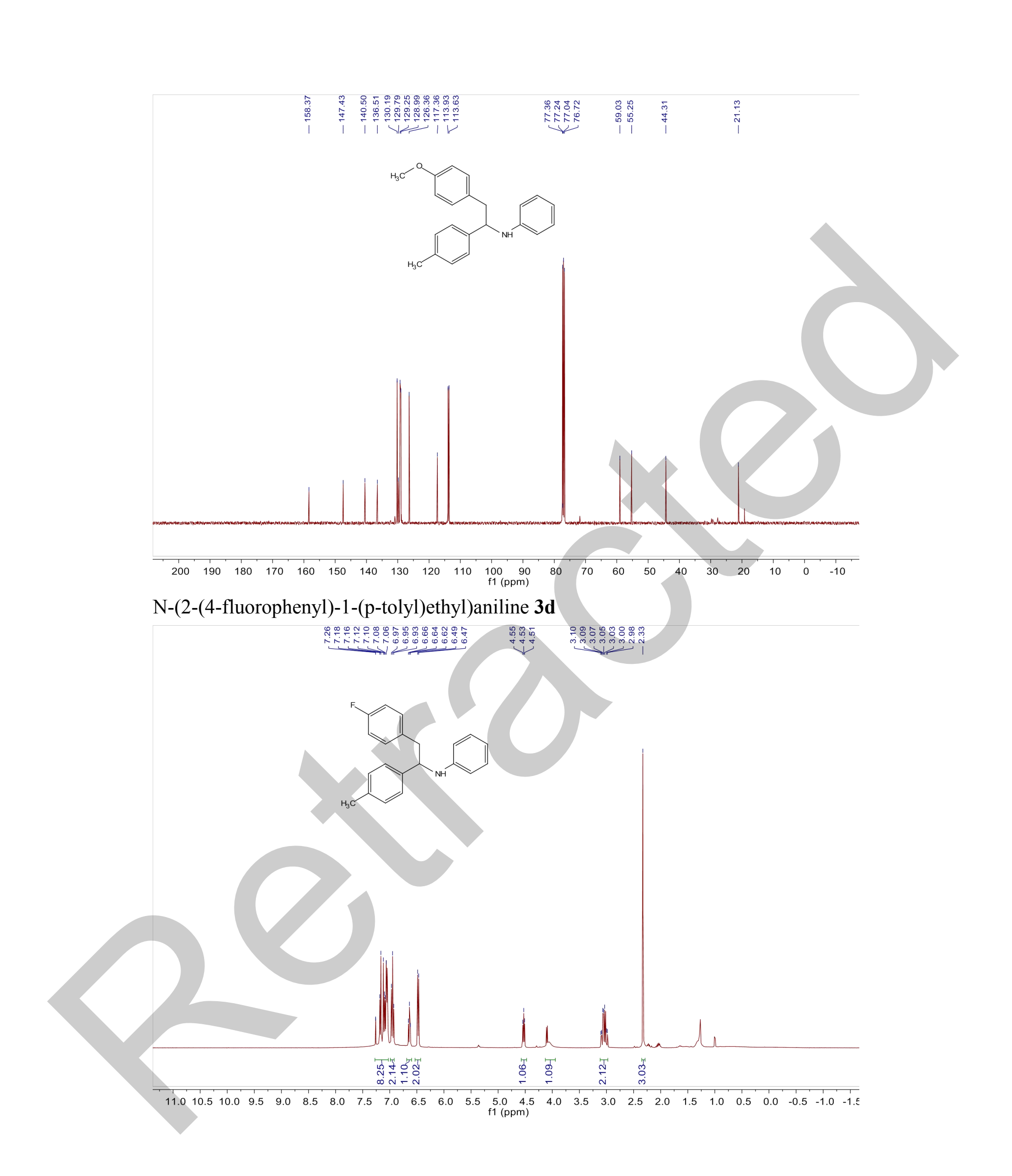




$$
\text { 29 }
$$




$$
\text { Pent }
$$




$$
\frac{11}{p^{e^{2}}}
$$




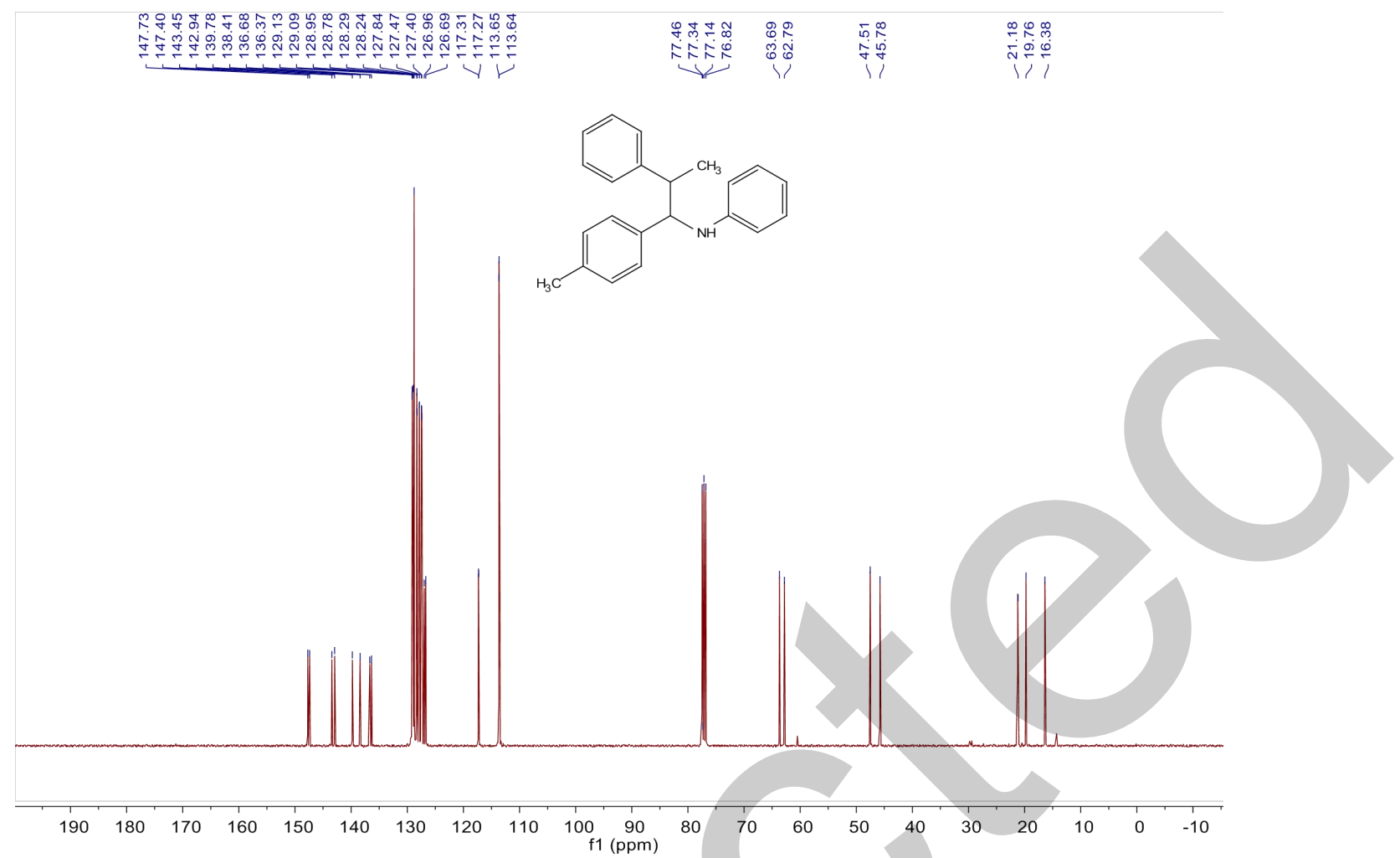

N-(2-(furan-2-yl)-1-(p-tolyl)ethyl)aniline $\mathbf{3 h}$



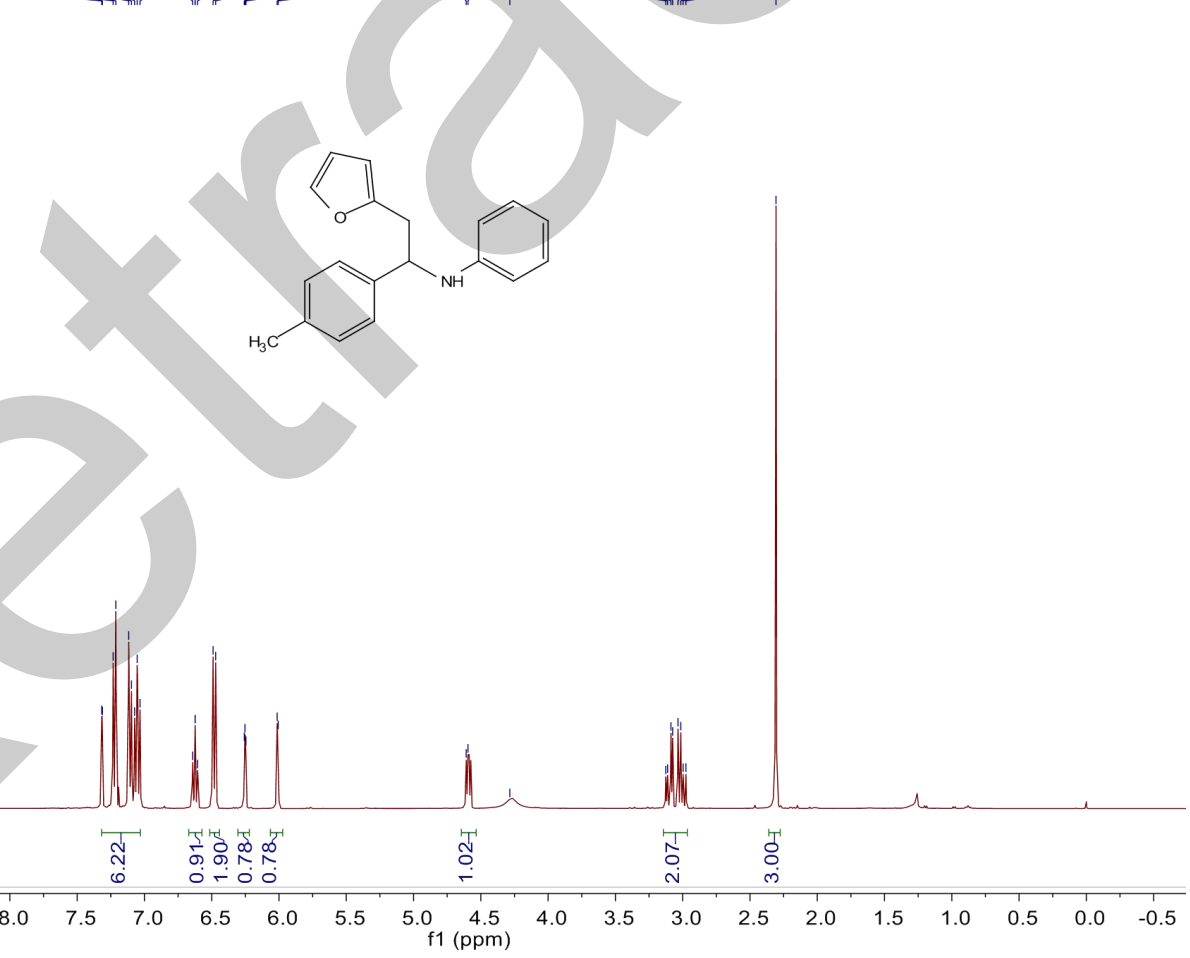




$$
\frac{e^{2}}{r^{2}}
$$




$$
\begin{aligned}
& \frac{a^{\infty}}{=x^{2}} \\
& \text { सiा }
\end{aligned}
$$



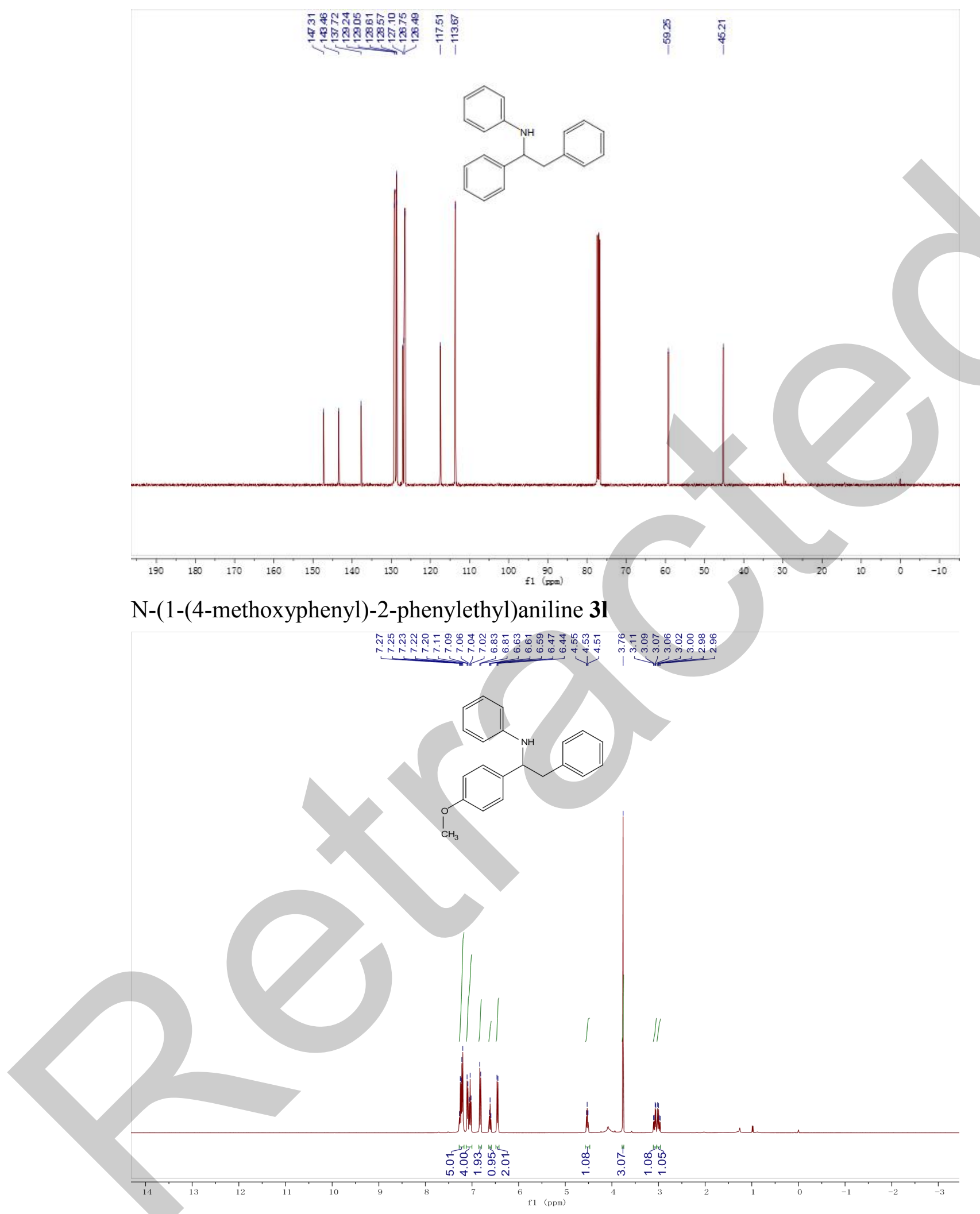

31 


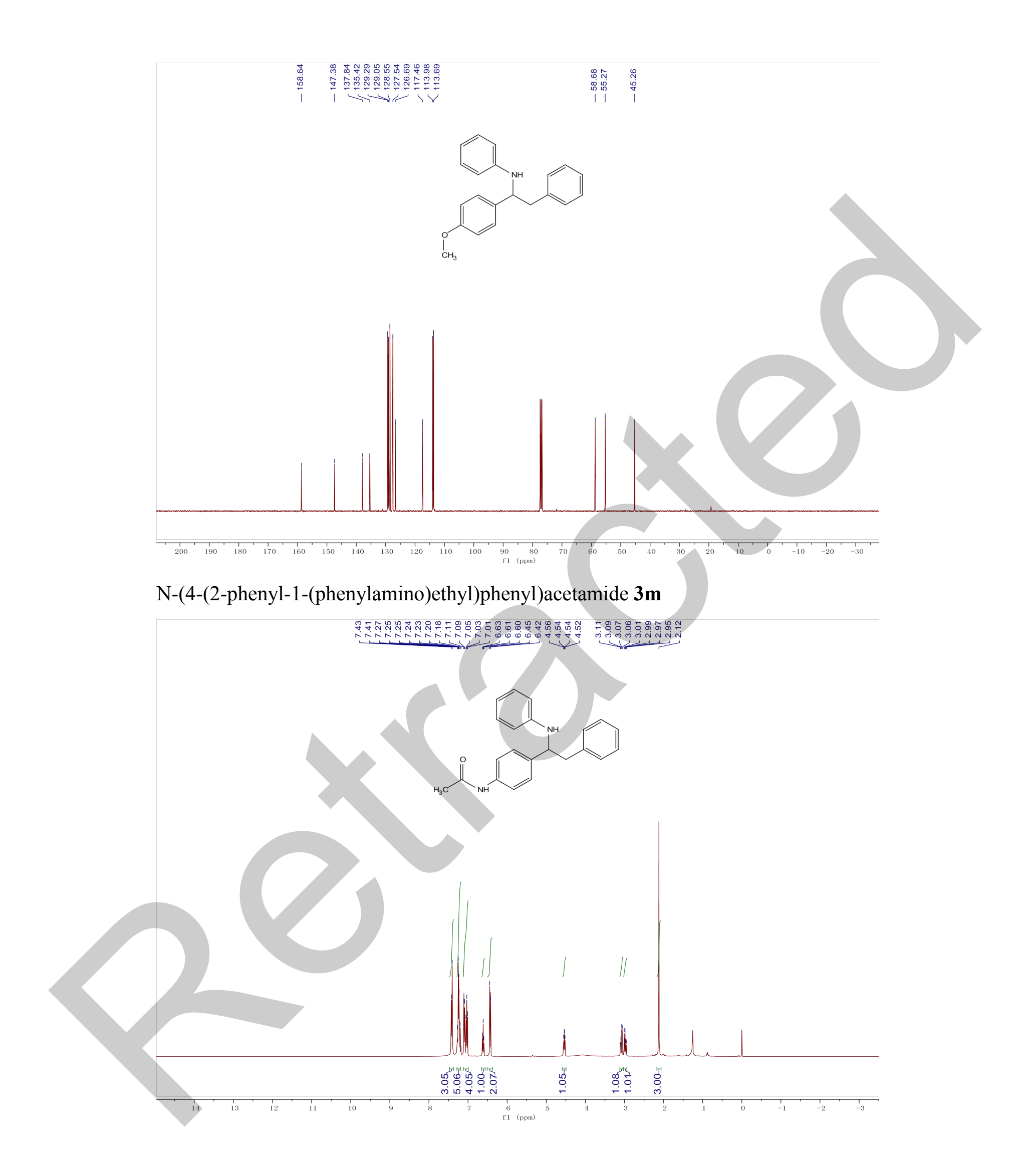




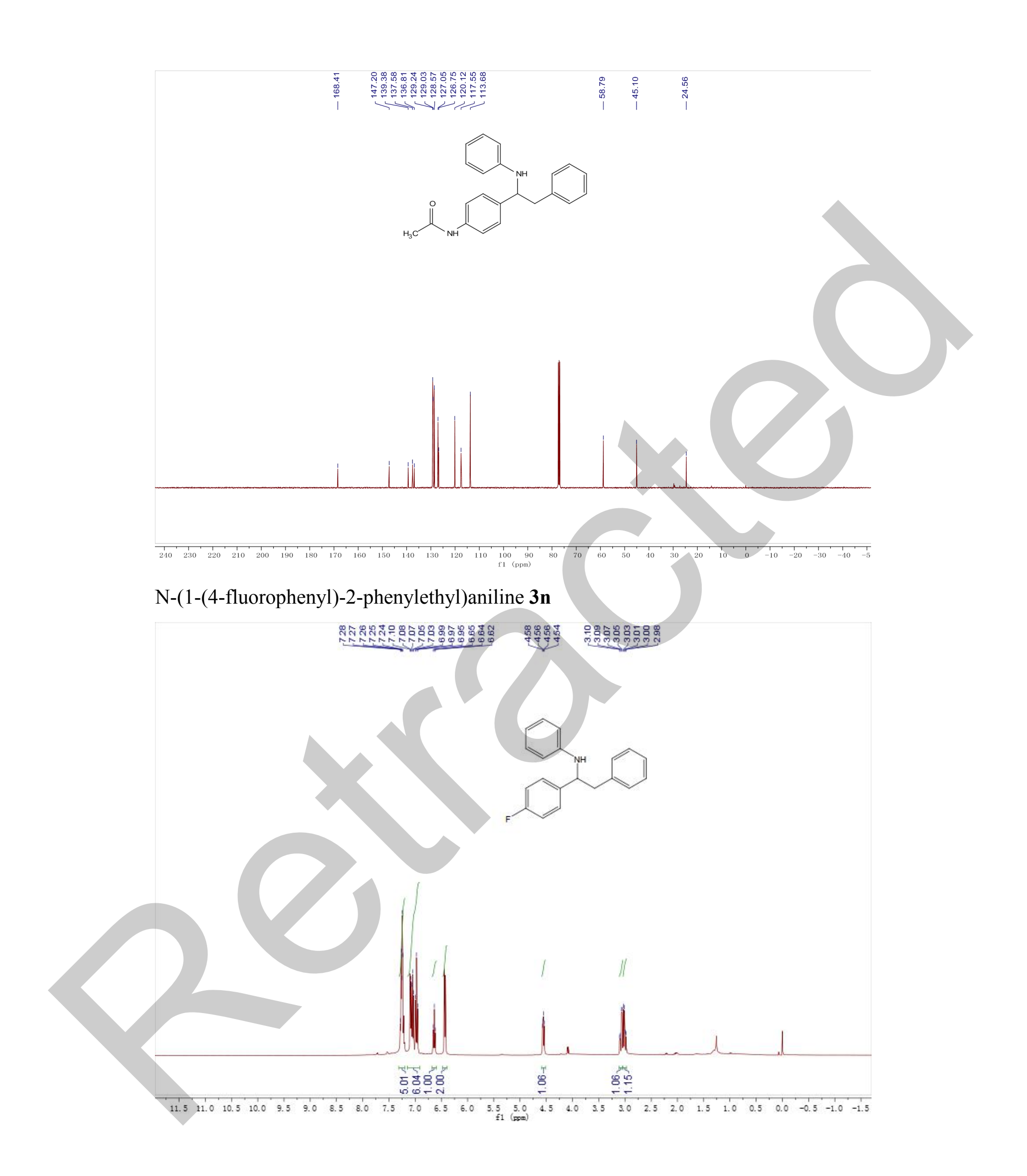




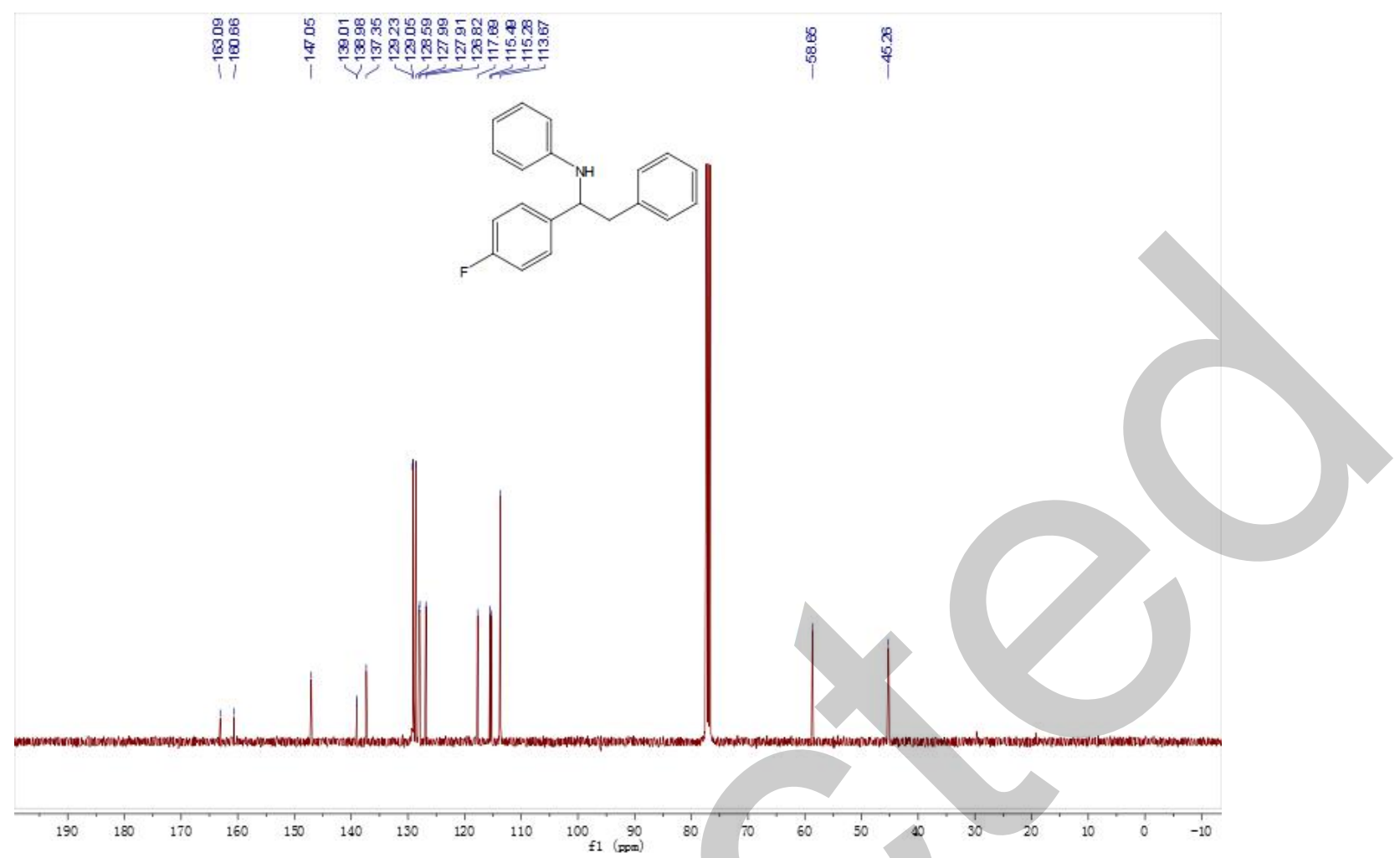

N-((4-bromophenyl)(phenyl)methyl)aniline 30

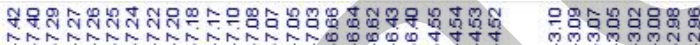
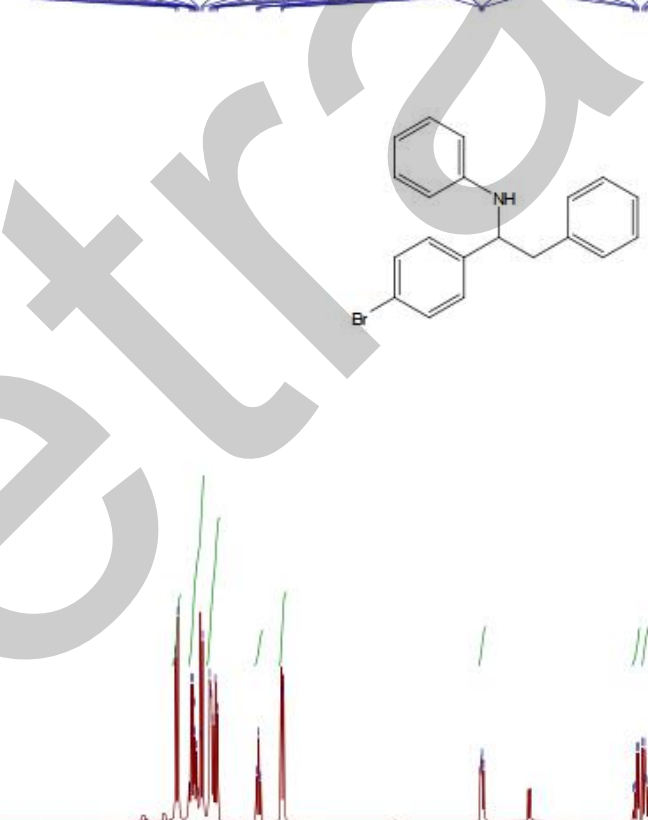

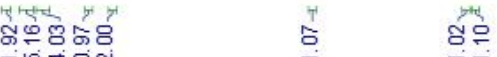

$\begin{array}{lllllllllllllllllllllllllllllllll}11.5 & 11.0 & 10.5 & 10.0 & 9.5 & 9.0 & 8.5 & 8.0 & 7.5 & 7.0 & 6.5 & 6.0 & 5.5 & 5.0 & 4.5 & 4.0 & 3.5 & 3.0 & 2.5 & 2.0 & 1.5 & 1.0 & 0.5 & 0.0 & -0.5 & -1.0 & -1.5\end{array}$ 


$$
\frac{11 e^{2}}{-y^{2}}
$$




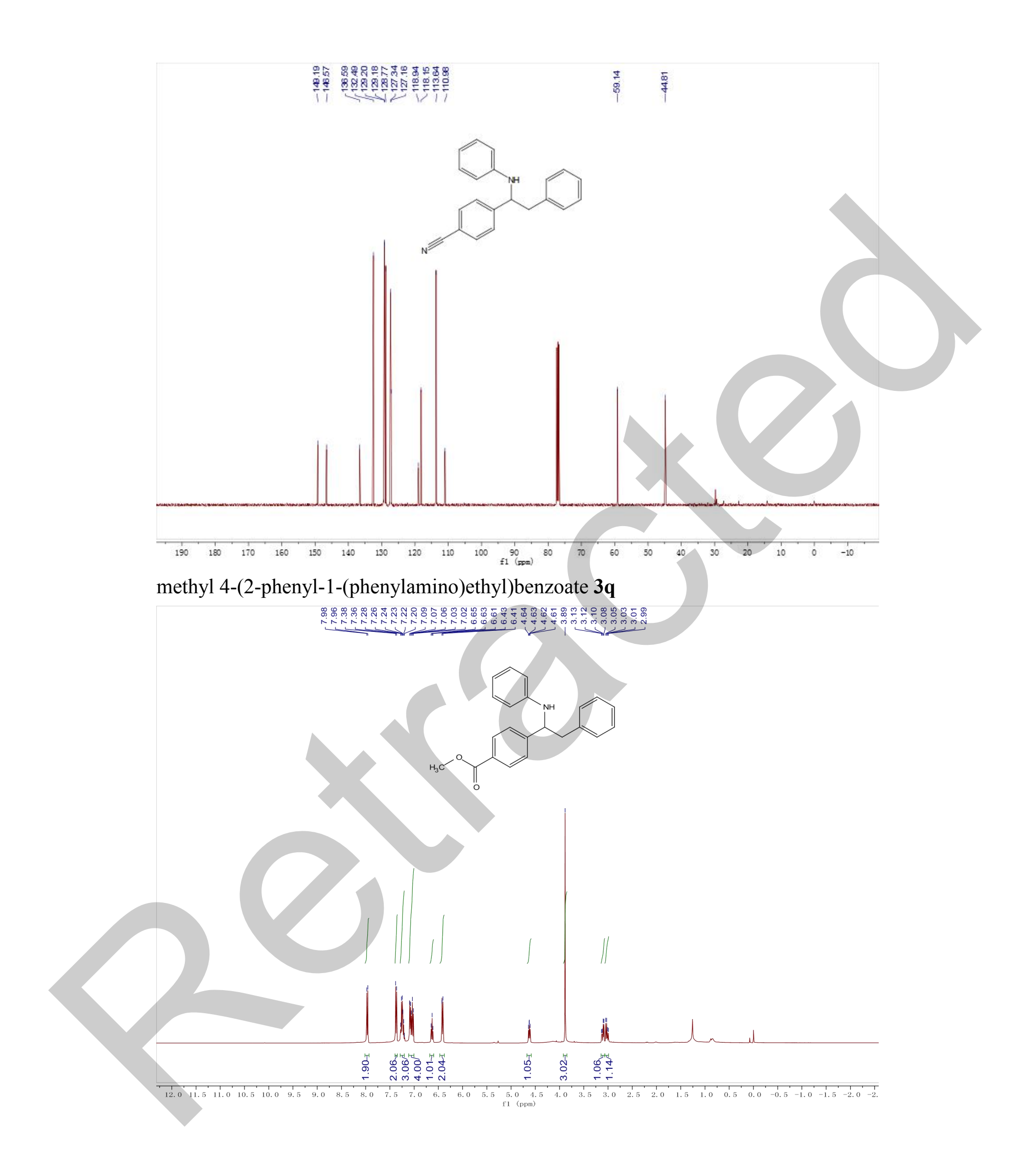




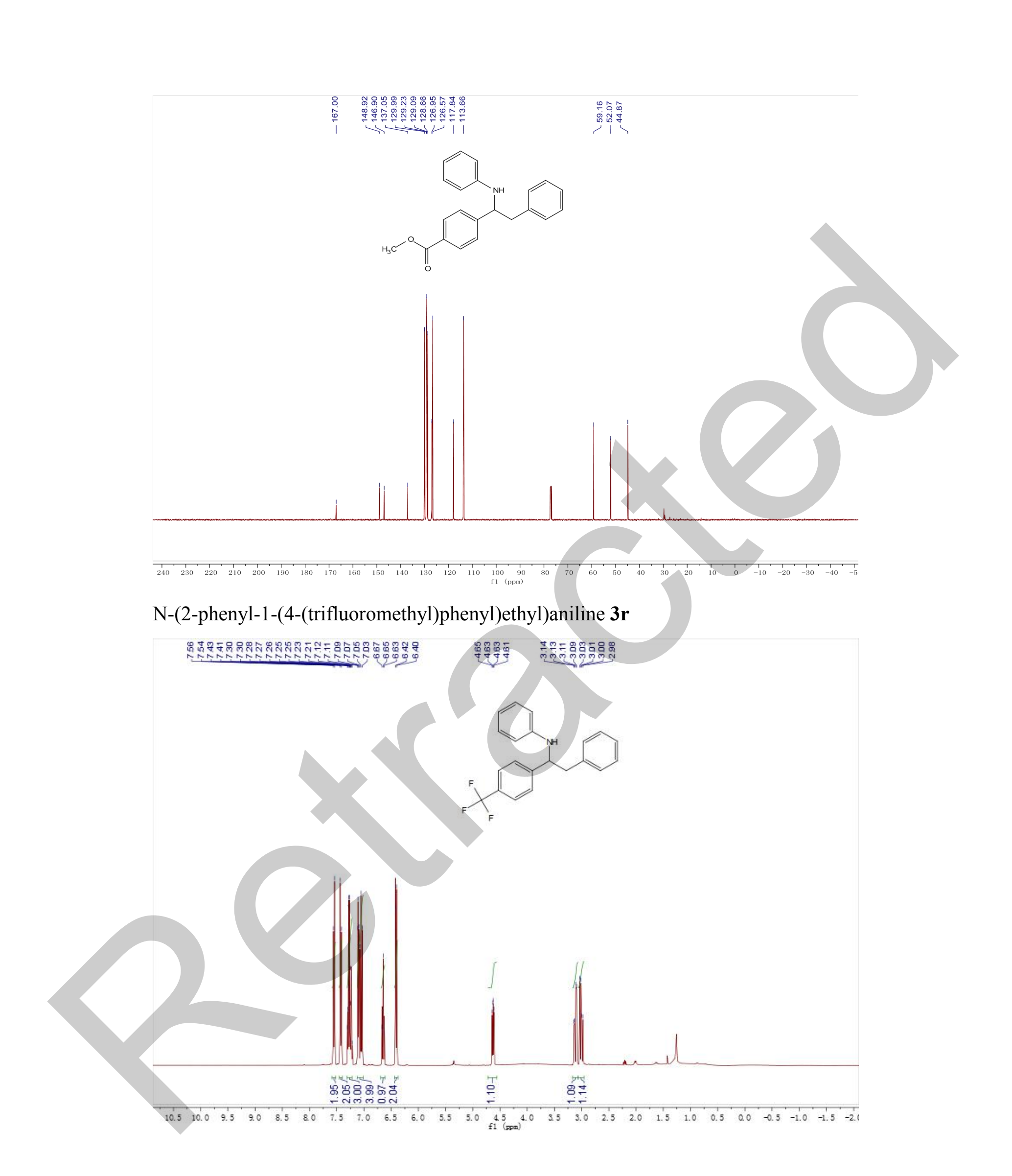




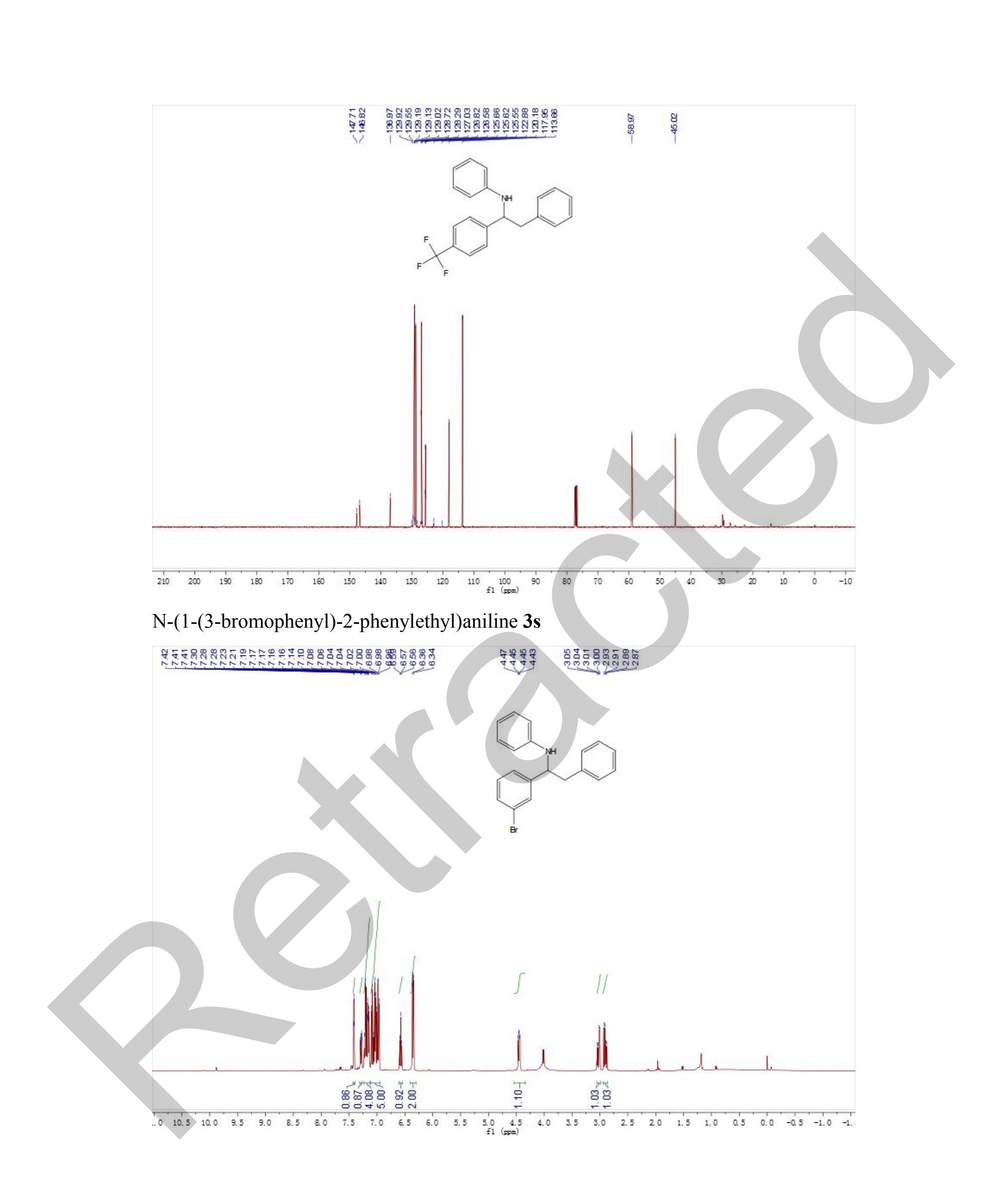




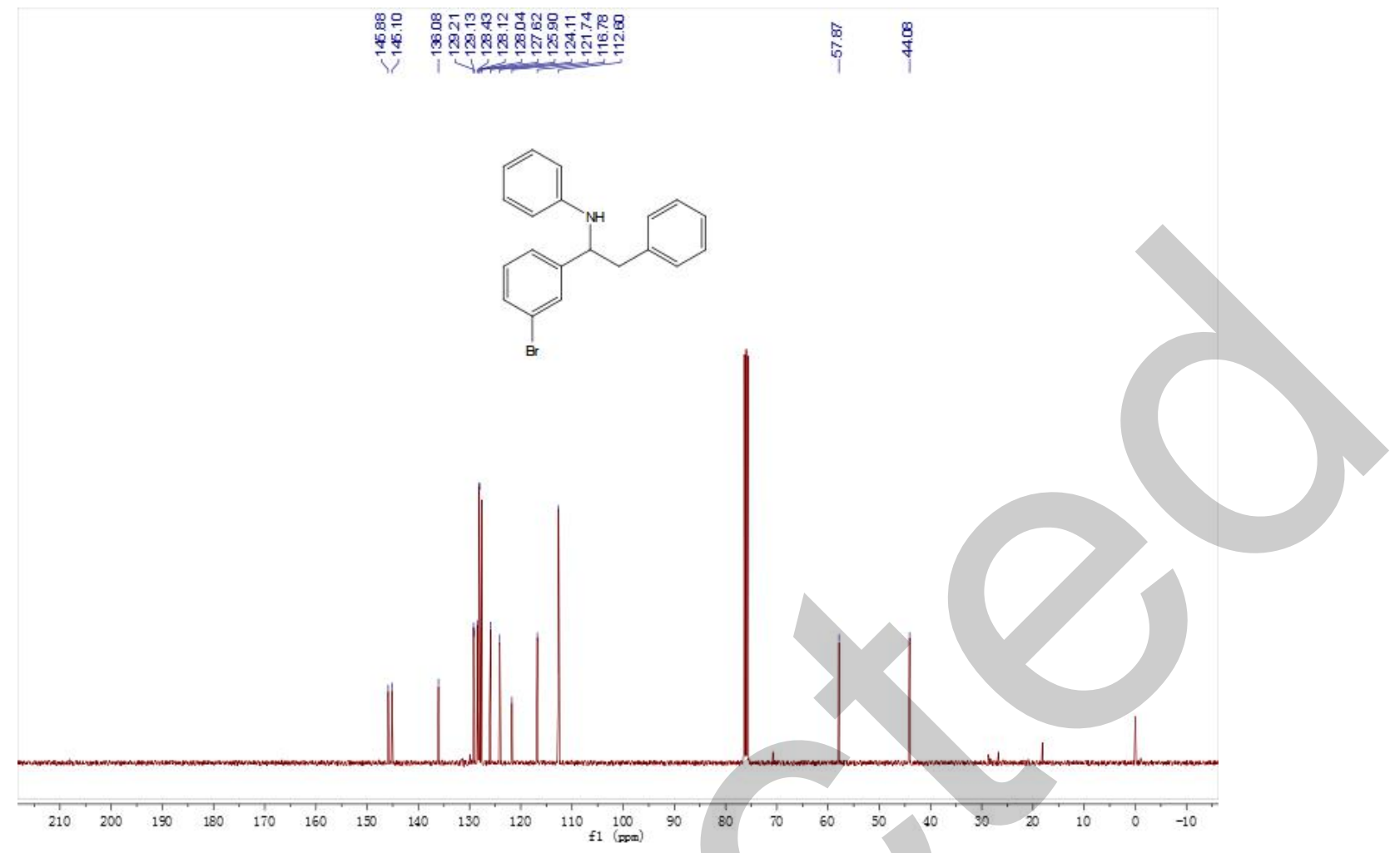

N-(1-(3-methoxyphenyl)-2-phenylethyl)aniline 3t

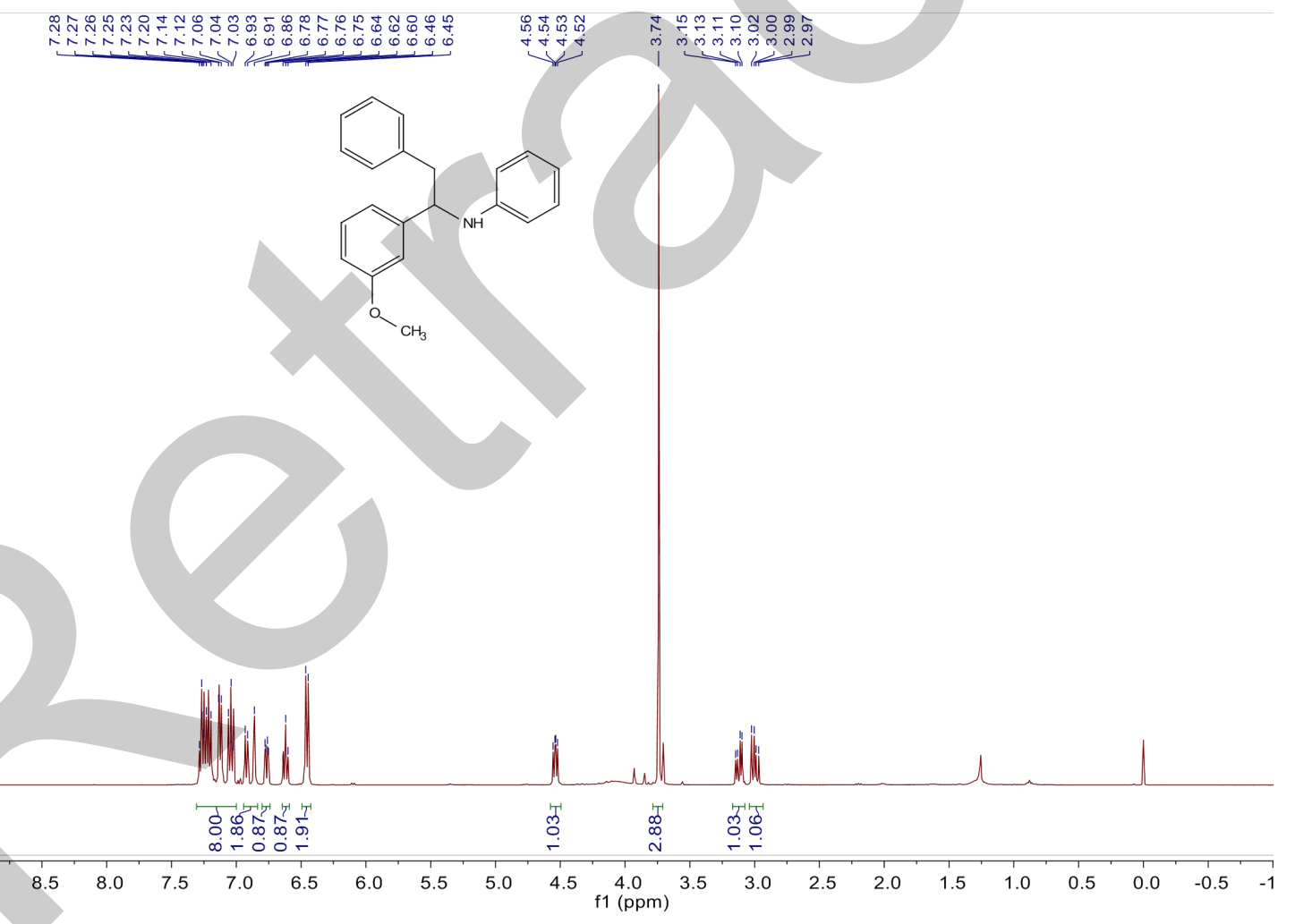




$$
\text { Rili. }
$$



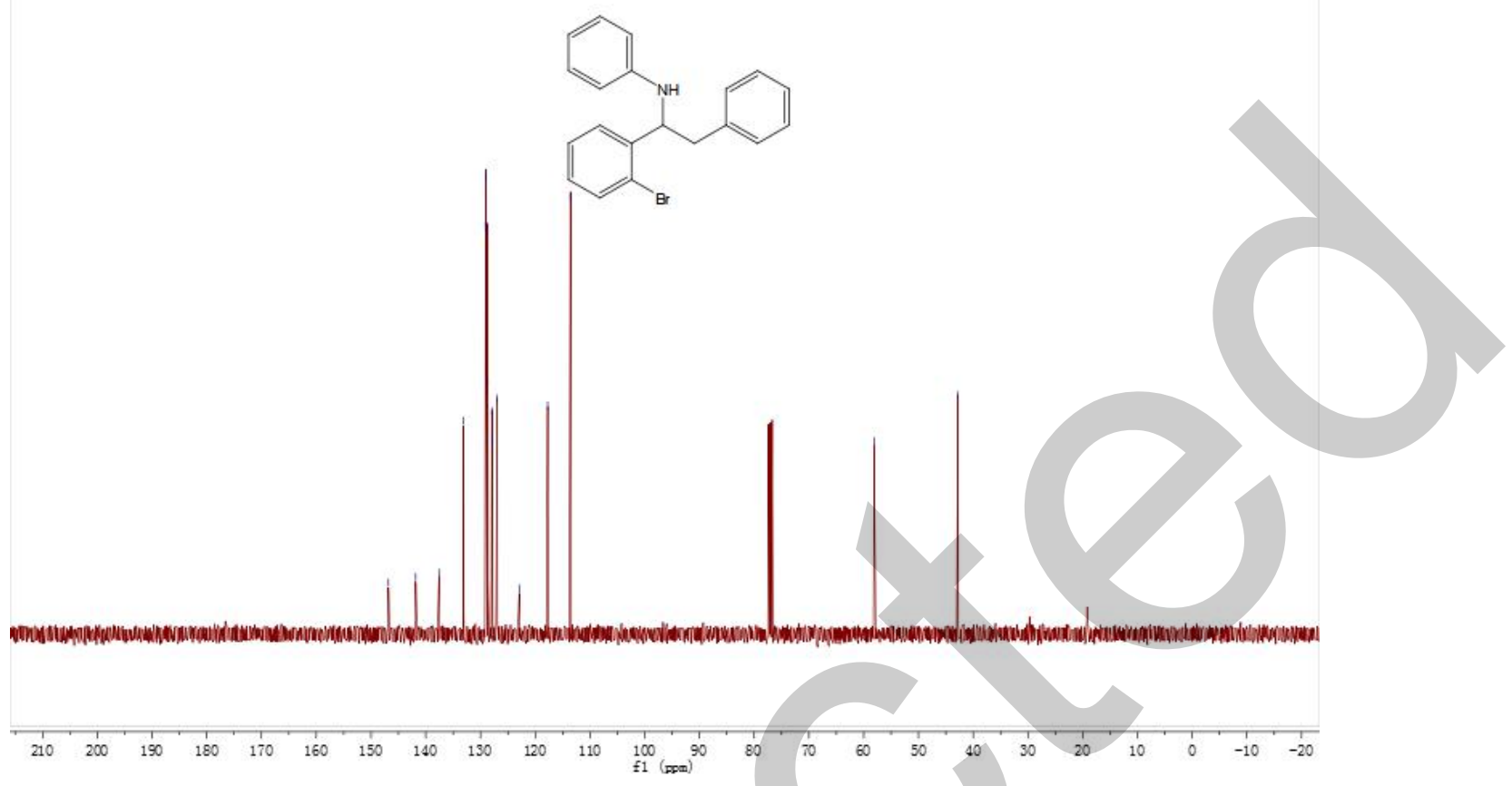

N-(1-(2-methoxyphenyl)-2-phenylethyl)aniline 3v

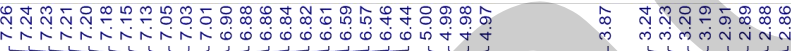






$$
e^{e^{x^{2}}}
$$




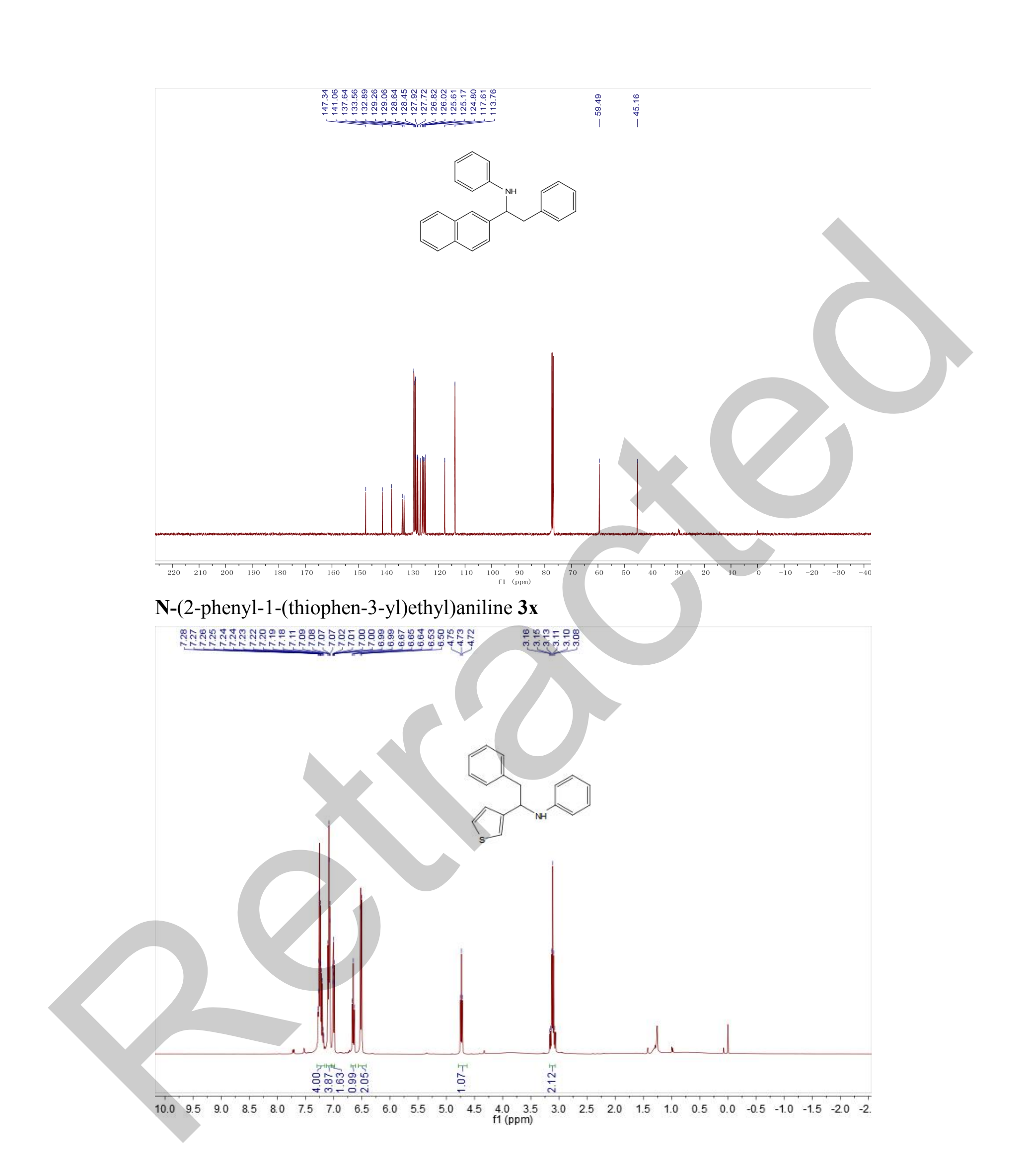




$$
\frac{1+e^{e^{d}}}{p^{e^{e^{2}}}}
$$




$$
\frac{e^{x^{2}}}{e^{20}}
$$




$$
\frac{e^{2}}{e^{x^{2}}}
$$




$$
\text { 29 }
$$




$$
\text { स- }
$$




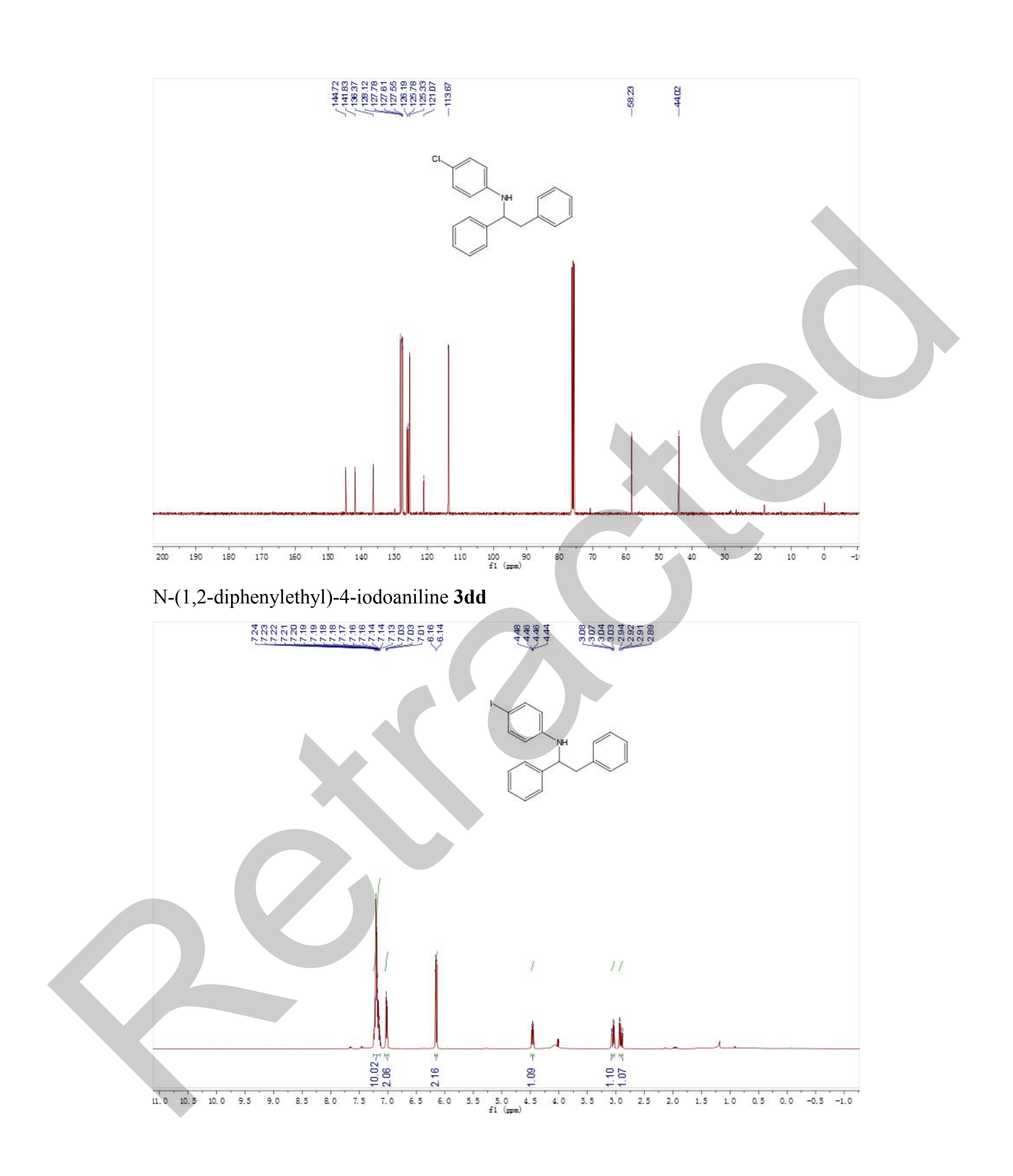




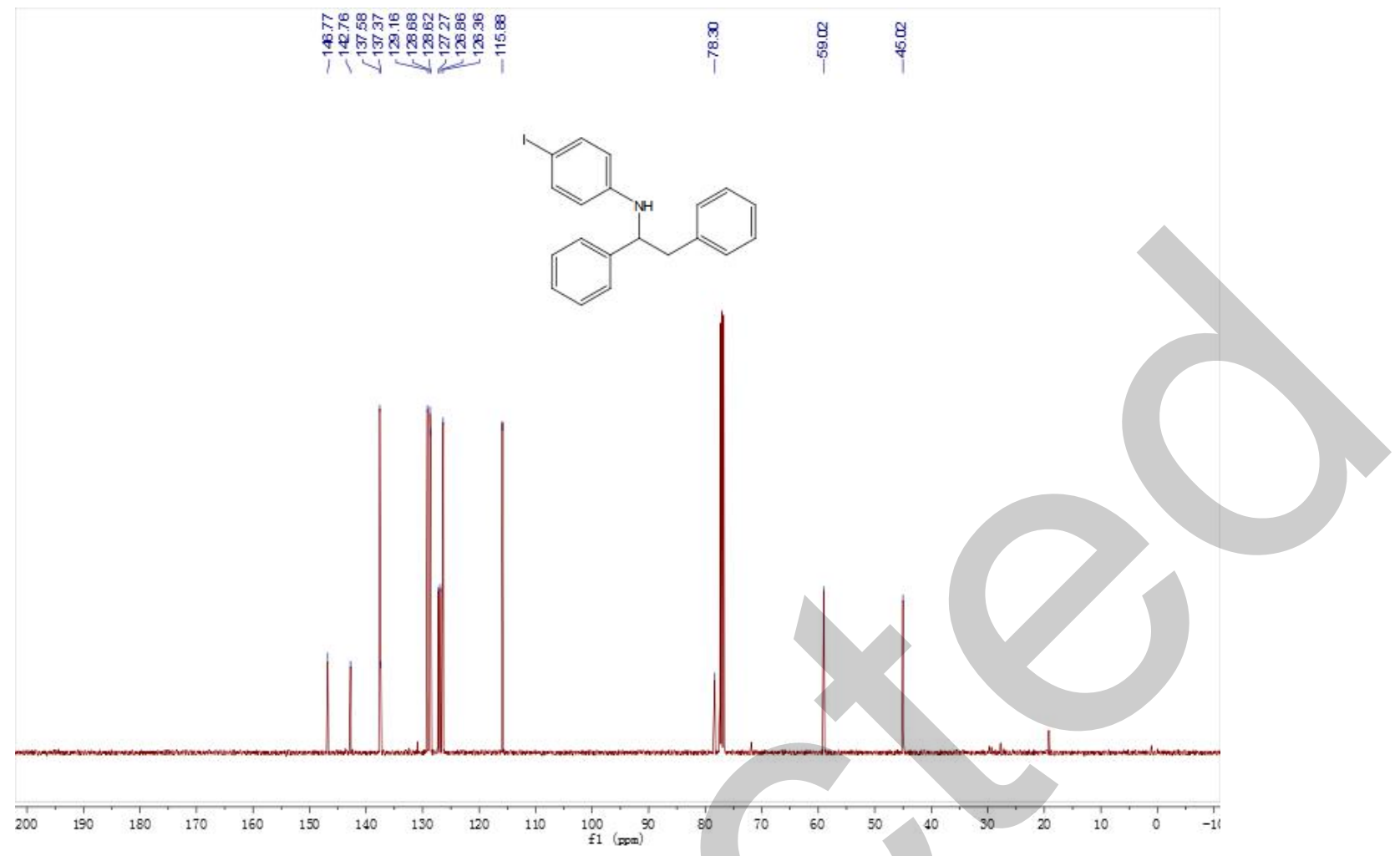

\section{References}

1. X. Hong, Hao Wang, B. Liu and B. Xu, Chem. Commun., 2014, 50, 14129-14132.

2. S. Murov, L., Handbook of Photochemistry, Marcel Dekker, New York, 1973. 